\title{
Electromagnetic Field Equation and Lorentz Gauge in Rindler Space-time
}

\author{
Sangwha-Yi* \\ Department of Mathematics, Taejon University, South Korea
}

In this paper, we derived electromagnetic field transformations and electromagnetic field equations of Maxwell in Rindler space-time in the context of general theory of relativity. We then treat the Lorentz gauge transformation and the Lorentz gauge fixing condition in Rindler space-time and obtained the transformation of differential operation, the electromagnetic 4-vector potential and the field. In addition, charge density and the electric current density in Rindler spacetime are derived. To view the invariance of the gauge transformation, gauge theory is applied to Maxwell equations in Rindler space-time. In Appendix A, we show that the electromagnetic wave function cannot exist in Rindler space-time. An important point we assert in this article is the uniqueness of the accelerated frame. It is because, in the accelerated frame, one can treat electromagnetic field equations.

\section{Introduction}

In 2007, del Castillio and Sanchez [1] discovered Maxwell equations in vacuum in a uniformly accelerated frame and Maluf and Faria [2] derived electromagnetic field transformations in Rindler space-time in 2011 [3]. They used Maxwell equations for gravity field but we disagree with their approach because Maxwell equations for a uniformly accelerated frame have to be treated in flat Minkowski space-time and not in the curved space-time, which implies the presence of gravitational field.

In this work, our aim is to find electromagnetic field equations in Rindler space-time, also in vacuum, but not in vacuum of the general relativity theory. In Sec. 2, after working out electromagnetic field equations in Rindler space-time, we derive the Lorentz gauge transformation and the Lorentz fixing condition, in addition to transformations for electromagnetic 4-vector potential in Rindler space-time. In Sec. 3, we define the electromagnetic field in Rindler space-time and we find the transformation of the electro-magnetic field. In Sec. 4, we obtain the electro-magnetic field equation in Rindler space-time and apply the gauge theory to Maxwell equations (worked out in earlier sections) in Rindler space-time for viewing the invariants of the gauge transformation.

We think it is important to know the electromagnetic wave function (radiation) in Rindler space-time but it is known that it does not satisfy electro-magnetic wave equation mathematically (see Appendix A). Hence according to our arguments, many results published during the period 2007 - 2011 (see Refs. [1] and [2]), especially the computation of electro-magnetic wave function, were incorrect. However, we do understand that electromagnetic wave function can exist in inertial frame as shown by Maxwell and Einstein.

\section{Transformation of the Electro-magnetic 4-vector Potential, Lorentz Gauge Transformation and Lorentz Gauge Fixing Condition}

The Rindler coordinate transformation is

$$
\begin{aligned}
& c t=\left(\frac{c^{2}}{a_{0}}+\xi^{1}\right) \sinh \left(\frac{a_{0} \xi^{0}}{c}\right) \\
& x=\left(\frac{c^{2}}{a_{0}}+\xi^{1}\right) \cosh \left(\frac{a_{0} \xi^{0}}{c}\right)-\frac{c^{2}}{a_{0}}, y=\xi^{2}, z=\xi^{3}
\end{aligned}
$$

The tetrad $e^{a} \mu$ is (see Refs. [4,5])

$$
\begin{aligned}
d \tau^{2} & =d t^{2}-\frac{1}{c^{2}}\left[d x^{2}+d y^{2}+d z^{2}\right] \\
& =-\frac{1}{c^{2}} \eta_{a b} \frac{\partial x^{a}}{\partial \xi^{\mu}} \frac{\partial x^{b}}{\partial \xi^{v}} d \xi^{\mu} d \xi^{v} \\
& =-\frac{1}{c^{2}} \eta_{a b} e^{a}{ }_{\mu} e^{b}{ }_{v} d \xi^{\mu} d \xi^{v}=-\frac{1}{c^{2}} g_{\mu v} d \xi^{\mu} d \xi^{v},
\end{aligned}
$$

"sangwha1@nate.com 


$$
\begin{gathered}
e^{a}{ }_{\mu}=\frac{\partial x^{a}}{\partial \xi^{\mu}} \\
e^{\alpha}{ }_{0}\left(\xi^{0}\right)=\frac{\partial x^{\alpha}}{c \partial \xi^{0}}=\left(\left(1+\frac{a_{0}}{c^{2}} \xi^{1}\right) \cosh \left(\frac{a_{0} \xi^{0}}{c}\right),\right. \\
\left.\left(1+\frac{a_{0}}{c^{2}} \xi^{1}\right) \sinh \left(\frac{a_{0} \xi^{0}}{c}\right), 0,0\right)
\end{gathered}
$$

The orientation of $y$-axis and $z$-axis is given as

$$
\begin{aligned}
& e_{2}^{\alpha}\left(\xi^{0}\right)=\frac{\partial x^{\alpha}}{\partial \xi^{2}}=(0,0,1,0) \\
& e_{3}^{\alpha}\left(\xi^{0}\right)=\frac{\partial x^{\alpha}}{\partial \xi^{3}}=(0,0,0,1)
\end{aligned}
$$

Where, the unit vector $e^{\alpha}{ }_{1}\left(\xi^{0}\right)$ is given as

$$
e^{\alpha}{ }_{1}\left(\xi^{0}\right)=\frac{\partial x^{\alpha}}{\partial \xi^{1}}=\left(\sinh \left(\frac{a_{0} \xi^{0}}{c}\right), \cosh \left(\frac{a_{0} \xi^{0}}{c}\right), 0,0\right)
$$

Therefore,

$$
\begin{aligned}
& c d t=c \cosh \left(\frac{a_{0} \xi^{0}}{c}\right) d \xi^{0}\left(1+\frac{a_{0}}{c^{2}} \xi^{1}\right)+\sinh \left(\frac{a_{0} \xi^{0}}{c}\right) d \xi^{1} \\
& d x=c \sinh \left(\frac{a_{0} \xi^{0}}{c}\right) d \xi^{0}\left(1+\frac{a_{0}}{c^{2}} \xi^{1}\right)+\cosh \left(\frac{a_{0} \xi^{0}}{c}\right) d \xi^{1}, \\
& d y=d \xi^{2}, d z=d \xi^{3}
\end{aligned}
$$

Now, the vector transformation is

$$
V^{\prime \mu}=\frac{\partial x^{\prime \mu}}{\partial x^{\alpha}} V^{\alpha}, U_{\mu}^{\prime}=\frac{\partial x^{\alpha}}{\partial x^{\prime \mu}} U_{\alpha}
$$

Therefore, the transformation of the electromagnetic 4-vector potential $(\phi, \vec{A})=A^{\alpha}$ is given by the following equations:

$$
A^{\alpha}=\frac{\partial x^{\alpha}}{\partial x^{\prime \mu}} A^{\prime \mu}=\frac{\partial x^{\alpha}}{\partial \xi^{\mu}} A_{\xi}{ }^{\mu}=e^{\alpha}{ }_{\mu} A_{\xi}{ }^{\mu}, \quad e^{\alpha}{ }_{\mu}=\frac{\partial x^{\alpha}}{\partial \xi^{\mu}}
$$

The transformation of differential coordinates is

$$
d x^{\alpha}=\frac{\partial x^{\alpha}}{\partial x^{\prime \mu}} d x^{\prime \mu}=\frac{\partial x^{\alpha}}{\partial \xi^{\mu}} d \xi^{\mu}=e^{\alpha}{ }_{\mu} d \xi^{\mu}, e^{\alpha}{ }_{\mu}=\frac{\partial x^{\alpha}}{\partial \xi^{\mu}}
$$

And, the equation of electro-magnetic 4-vector potential $(\phi, \vec{A})=A^{\alpha}$ is

$$
\begin{aligned}
& \left(\frac{1}{c^{2}} \frac{\partial^{2}}{\partial t^{2}}-\nabla^{2}\right) \phi=4 \pi \rho \\
& \left(\frac{1}{c^{2}} \frac{\partial^{2}}{\partial t^{2}}-\nabla^{2}\right) \vec{A}=\frac{4 \pi}{c} \vec{j} \\
& \text { 4-vector }(c \rho, \vec{j})=\rho_{0} \frac{d x^{\alpha}}{d \tau}
\end{aligned}
$$

Lorentz gauge transformation in Rindler space-time is given by

$$
A^{\mu} \rightarrow A^{\mu}+\partial^{\mu} \Lambda=A^{\mu}+g^{\mu v} \partial_{v} \Lambda
$$

Where, $\Lambda$ is a scalar function.

$$
\begin{aligned}
& g^{00}=-\frac{1}{\left(1+\frac{a_{0} \xi^{1}}{c^{2}}\right)^{2}}, g^{11}=g^{22}=g^{33}=1 \\
& \phi_{\xi} \rightarrow \phi_{\xi}-\frac{1}{c} \frac{\partial \Lambda}{\partial \xi^{0}} \frac{1}{\left(1+\frac{a_{0} \xi^{1}}{c^{2}}\right)^{2}}, \vec{A}_{\xi} \rightarrow \vec{A}_{\xi}+\vec{\nabla}_{\xi} \Lambda
\end{aligned}
$$

Here, $\Lambda$ is a scalar function.

Therefore, the Lorentz gauge in Rindler spacetime can be written as

$$
\begin{aligned}
& A_{; \mu}^{\mu}=\frac{\partial A^{\mu}}{\partial \xi^{\mu}}+\Gamma_{\mu \rho}^{\mu} A^{\rho}, \\
& \Gamma_{\mu \rho}^{\mu}=\Gamma_{01}^{0}=\frac{1}{2} g^{00}\left(\frac{\partial g_{00}}{\partial \xi^{1}}\right)=\frac{a_{0}}{c^{2}} \frac{1}{\left(1+\frac{a_{0}}{c^{2}} \xi^{1}\right)} \\
& g^{00}=-\frac{1}{\left(1+\frac{a_{0} \xi^{1}}{c^{2}}\right)^{2}}, g^{11}=g^{22}=g^{33}=1 \\
& 0=\frac{\partial \phi}{c \partial t}+\vec{\nabla} \cdot \vec{A}=\frac{\partial \phi_{\xi}}{c \partial \xi^{0}}+\vec{\nabla}_{\xi} \cdot \vec{A}_{\xi}+\frac{A_{\xi^{1}} a_{0}}{c^{2}} \frac{1}{\left(1+\frac{a_{0} \xi^{1}}{c^{2}}\right)}
\end{aligned}
$$

Hence, Lorentz gauge transformation and Lorentz gauge in Rindler space-time are as follows:

$$
\begin{aligned}
A_{; \mu}^{\mu}= & \frac{\partial A^{\mu}}{\partial \xi^{\mu}}+\Gamma^{\mu}{ }_{\mu \rho} A^{\rho} \rightarrow \\
& \partial_{\mu}\left(A^{\mu}+g^{\mu v} \partial_{v} \Lambda\right)+\Gamma_{01}^{0}\left(A^{1}+\frac{\partial \Lambda}{\partial \xi^{1}}\right)
\end{aligned}
$$




$$
\begin{gathered}
=\partial_{\mu} A^{\mu}+\left(g^{\mu v} \partial_{\mu} \partial_{v}\right) \Lambda+\Gamma^{0}{ }_{01}\left(A^{1}+\frac{\partial \Lambda}{\partial \xi^{1}}\right) \\
0=\frac{\partial \phi_{\xi}}{c \partial \xi^{0}}+\vec{\nabla}_{\xi} \cdot \vec{A}_{\xi}+\frac{A_{\xi^{1}} a_{0}}{c^{2}} \frac{1}{\left(1+\frac{a_{0} \xi^{1}}{c^{2}}\right)} \\
\rightarrow \frac{1}{c} \frac{\partial \phi_{\xi}}{\partial \xi^{0}}+\vec{\nabla}_{\xi} \cdot \vec{A}_{\xi}-\left[\frac{1}{c^{2}} \frac{1}{\left(1+\frac{a_{0} \xi^{1}}{c^{2}}\right)^{2}}\left(\frac{\partial}{\partial \xi^{0}}\right)^{2}-\nabla_{\xi}{ }^{2}\right] \Lambda \\
+\frac{A_{\xi^{1}} a_{0}}{c^{2}} \frac{1}{\left(1+\frac{a_{0}}{c^{2}} \xi^{1}\right)}+\frac{\partial \Lambda}{\partial \xi^{1}} \frac{a_{0}}{c^{2}} \frac{1}{\left(1+\frac{a_{0} \xi^{1}}{c^{2}}\right)}=0(11-\mathrm{i})
\end{gathered}
$$

Hence, one can obtain Lorentz gauge fixing condition in Rindler space-time as

$$
\left[\frac{1}{c^{2}} \frac{1}{\left(1+\frac{a_{0} \xi^{1}}{c^{2}}\right)^{2}}\left(\frac{\partial}{\partial \xi^{0}}\right)^{2}-\nabla_{\xi}^{2}\right] \Lambda
$$

$$
-\frac{\partial \Lambda}{\partial \xi^{1}} \frac{a_{0}}{c^{2}} \frac{1}{\left(1+\frac{a_{0} \xi^{1}}{c^{2}}\right)}=0
$$

From the result obtained above, we found the transformation of the electro-magnetic 4-vector potential $(\phi, \vec{A})$ in an inertial frame and the electro-magnetic 4-vector potential $\left(\phi_{\xi}, \vec{A}_{\xi}\right)$ in a uniformly accelerated frame, which can be written as

$$
\begin{aligned}
& \phi=\cosh \left(\frac{a_{0} \xi^{0}}{c}\right)\left(1+\frac{a_{0}}{c^{2}} \xi^{1}\right) \phi_{\xi}+\sinh \left(\frac{a_{0} \xi^{0}}{c}\right) A_{\xi^{1}} \\
& A_{x}=\sinh \left(\frac{a_{0} \xi^{0}}{c}\right)\left(1+\frac{a_{0}}{c^{2}} \xi^{1}\right) \phi_{\xi}+\cosh \left(\frac{a_{0} \xi^{0}}{c}\right) A_{\xi^{1}} \\
& A_{y}=A_{\xi^{2}}, A_{z}=A_{\xi^{3}}
\end{aligned}
$$

If we take the matrix of the transformation in the differential coordinate, then we have

$$
\left(\begin{array}{l}
c d t \\
d x \\
d y \\
d z
\end{array}\right)=\left(\begin{array}{cccc}
\cosh \left(\frac{a_{0} \xi^{0}}{c}\right)\left(1+\frac{a_{0} \xi^{1}}{c^{2}}\right) & \sinh \left(\frac{a_{0} \xi^{0}}{c}\right) & 0 & 0 \\
\sinh \left(\frac{a_{0} \xi^{0}}{c}\right)\left(1+\frac{a_{0} \xi^{1}}{c^{2}}\right) & \cosh \left(\frac{a_{0} \xi^{0}}{c}\right) & 0 & 0 \\
0 & 0 & 1 & 0 \\
0 & 0 & 0 & 1
\end{array}\right)\left(\begin{array}{c}
c d \xi^{0} \\
d \xi^{1} \\
d \xi^{2} \\
d \xi^{3}
\end{array}\right)=A\left(\begin{array}{c}
c d \xi^{0} \\
d \xi^{1} \\
d \xi^{2} \\
d \xi^{3}
\end{array}\right)
$$

The inverse matrix of Eqn. (14) [12] and [14] can be obtained as

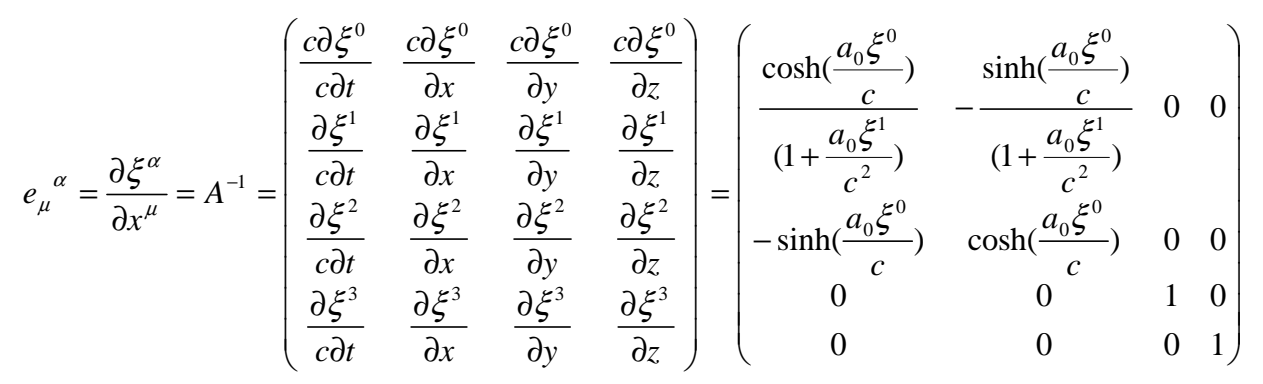

Hence, we can obtain the matrix of transformation of differential operation as 


$$
\left(\begin{array}{c}
\frac{1}{c} \frac{\partial}{\partial t} \\
\frac{\partial}{\partial x} \\
\frac{\partial}{\partial y} \\
\frac{\partial}{\partial z}
\end{array}\right)=\left(A^{-1}\right)^{T}\left(\begin{array}{c}
\frac{1}{c} \frac{\partial}{\partial \xi^{0}} \\
\frac{\partial}{\partial \xi^{1}} \\
\frac{\partial}{\partial \xi^{2}} \\
\frac{\partial}{\partial \xi^{3}}
\end{array}\right)=\left(A^{T}\right)^{-1}\left(\begin{array}{c}
\frac{1}{c} \frac{\partial}{\partial \xi^{0}} \\
\frac{\partial}{\partial \xi^{1}} \\
\frac{\partial}{\partial \xi^{2}} \\
\frac{\partial}{\partial \xi^{3}}
\end{array}\right)=\left(\begin{array}{rrrr}
\frac{\cosh \left(\frac{a_{0} \xi^{0}}{c}\right)}{\left(1+\frac{a_{0} \xi^{1}}{c^{2}}\right)} & -\sinh \left(\frac{a_{0} \xi^{0}}{c}\right) & 0 & 0 \\
\left.-\frac{a_{0} \xi^{0}}{c}\right) & & \\
\left.-\frac{a_{0} \xi^{1}}{c^{2}}\right) & \cosh \left(\frac{a_{0} \xi^{0}}{c}\right) & 0 & 0 \\
0 & 0 & 1 & 0 \\
0 & 0 & 0 & 1
\end{array}\right)\left(\begin{array}{c}
\frac{1}{c} \frac{\partial}{\partial \xi^{0}} \\
\frac{\partial}{\partial \xi^{1}} \\
\frac{\partial}{\partial \xi^{2}} \\
\frac{\partial}{\partial \xi^{3}}
\end{array}\right)
$$

Therefore, the transformation of differential operation is

$$
\begin{aligned}
\frac{1}{c} \frac{\partial}{\partial t} & =\frac{c \partial \xi^{0}}{c \partial t} \frac{1}{c} \frac{\partial}{\partial \xi^{0}}+\frac{\partial \xi^{1}}{c \partial t} \frac{\partial}{\partial \xi^{1}} \\
& =\frac{\cosh \left(\frac{a_{0} \xi^{0}}{c}\right)}{\left(1+\frac{a_{0} \xi^{1}}{c^{2}}\right)} \frac{\partial}{c \partial \xi^{0}}-\sinh \left(\frac{a_{0} \xi^{0}}{c}\right) \frac{\partial}{\partial \xi^{1}} \\
\frac{\partial}{\partial x} & =\frac{c \partial \xi^{0}}{\partial x} \frac{1}{c} \frac{\partial}{\partial \xi^{0}}+\frac{\partial \xi^{1}}{\partial x} \frac{\partial}{\partial \xi^{1}} \\
& =-\frac{\sinh \left(\frac{a_{0} \xi^{0}}{c}\right)}{\left(1+\frac{a_{0} \xi^{1}}{c^{2}}\right)} \frac{\partial}{c \partial \xi^{0}}+\cosh \left(\frac{a_{0} \xi^{0}}{c}\right) \frac{\partial}{\partial \xi^{1}} \\
\frac{\partial}{\partial y}=\frac{\partial}{\partial \xi^{2}}, \frac{\partial}{\partial z} & =\frac{\partial}{\partial \xi^{3}}
\end{aligned}
$$

The above differential operation satisfies the following equations:

$$
\begin{gathered}
\frac{1}{c^{2}} \frac{\partial^{2}}{\partial t^{2}}-\nabla^{2}=\frac{1}{c^{2}\left(1+\frac{a_{0}}{c^{2}} \xi^{1}\right)^{2}}\left(\frac{\partial}{\partial \xi^{0}}\right)^{2}-\nabla_{\xi}^{2} \\
\vec{\nabla}=\left(\frac{\partial}{\partial x}, \frac{\partial}{\partial y}, \frac{\partial}{\partial z}\right), \quad \vec{\nabla}_{\xi}=\left(\frac{\partial}{\partial \xi^{1}}, \frac{\partial}{\partial \xi^{2}}, \frac{\partial}{\partial \xi^{3}}\right)
\end{gathered}
$$

\section{Electro-magnetic Field in the Rindler Space-time}

Given the electromagnetic field $(\vec{E}, \vec{B})$ in the inertial frame as

$$
\vec{E}=-\vec{\nabla} \phi-\frac{\partial \vec{A}}{c \partial t}, \vec{B}=\vec{\nabla} \times \vec{A}
$$

We need to perform computation in order to define the electro-magnetic field in Rindler space-time, which requires that we calculate electromagnetic field transformations in Rindler space-time. The computation is straightforward by using the electromagnetic 4-vector potential transformation, Eqn. (13) and the transformation of differential operation (Eqn. (17)). One therefore obtains

$$
\begin{aligned}
E_{x}= & -\frac{\partial \phi}{\partial x}-\frac{\partial A_{x}}{c \partial t} \\
= & -\left[-\frac{\sinh \left(\frac{a_{0} \xi^{0}}{c}\right)}{\left(1+\frac{a_{0} \xi^{1}}{c^{2}}\right)} \frac{\partial}{c \partial \xi^{0}}+\cosh \left(\frac{a_{0} \xi^{0}}{c}\right) \frac{\partial}{\partial \xi^{1}}\right] \cdot\left[\cosh \left(\frac{a_{0} \xi^{0}}{c}\right)\left(1+\frac{a_{0} \xi^{1}}{c^{2}}\right) \phi_{\xi}+\sinh \left(\frac{a_{0} \xi^{0}}{c}\right) A_{\xi^{1}}\right] \\
& -\left[\frac{\cosh \left(\frac{a_{0} \xi^{0}}{c}\right)}{\left(1+\frac{a_{0} \xi^{1}}{c^{2}}\right)} \frac{\partial}{c \partial \xi^{0}}-\sinh \left(\frac{a_{0} \xi^{0}}{c}\right) \frac{\partial}{\partial \xi^{1}}\right] \cdot\left[\sinh \left(\frac{a_{0} \xi^{0}}{c}\right)\left(1+\frac{a_{0} \xi^{1}}{c^{2}}\right) \phi_{\xi}+\cosh \left(\frac{a_{0} \xi^{0}}{c}\right) A_{\xi^{1}}\right] \\
= & -\frac{1}{\left(1+\frac{a_{0}}{c^{2}} \xi^{1}\right)} \frac{\partial A_{\xi^{1}}}{c \partial \xi^{0}}-\left(1+\frac{a_{0} \xi^{1}}{c^{2}}\right) \frac{\partial \phi_{\xi}}{\partial \xi^{1}}-2 \phi_{\xi} \frac{a_{0}}{c^{2}}
\end{aligned}
$$




$$
=-\frac{1}{\left(1+\frac{a_{0} \xi^{1}}{c^{2}}\right)} \frac{\partial}{\partial \xi^{1}}\left[\left(1+\frac{a_{0}}{c^{2}} \xi^{1}\right)^{2} \phi_{\xi}\right]-\frac{1}{\left(1+\frac{a_{0} \xi^{1}}{c^{2}}\right)} \frac{\partial A_{\xi^{1}}}{c \partial \xi^{0}}
$$

The $y$-component of the electric field in the inertial frame is given as

$$
\begin{aligned}
& E_{y}=-\frac{\partial \phi}{\partial y}-\frac{\partial A_{y}}{c \partial t}=-\frac{\partial}{\partial \xi^{2}}\left[\cosh \left(\frac{a_{0} \xi^{0}}{c}\right)\left(1+\frac{a_{0}}{c^{2}} \xi^{1}\right) \phi_{\xi}+\sinh \left(\frac{a_{0} \xi^{0}}{c}\right) A_{\xi^{1}}\right] \\
& -\left[\frac{\cosh \left(\frac{a_{0} \xi^{0}}{c}\right)}{\left(1+\frac{a_{0} \xi^{1}}{c^{2}}\right)} \frac{\partial}{c \partial \xi^{0}}-\sinh \left(\frac{a_{0} \xi^{0}}{c}\right) \frac{\partial}{\partial \xi^{1}}\right] A_{\xi^{2}} \\
& =-\left(1+\frac{a_{0} \xi^{1}}{c^{2}}\right) \cosh \left(\frac{a_{0} \xi^{0}}{c}\right) \frac{\partial \phi_{\xi}}{\partial \xi^{2}}-\frac{1}{\left(1+\frac{a_{0} \xi^{1}}{c^{2}}\right)} \cosh \left(\frac{a_{0} \xi^{0}}{c}\right) \frac{\partial A_{\xi^{2}}}{c \partial \xi^{0}}+\sinh \left(\frac{a_{0}}{c} \xi^{0}\right)\left[\frac{\partial A_{\xi^{2}}}{\partial \xi^{1}}-\frac{\partial A_{\xi^{1}}}{\partial \xi^{2}}\right] \\
& =\cosh \left(\frac{a_{0}}{c} \xi^{0}\right)\left[-\frac{1}{\left(1+\frac{a_{0}}{c^{2}} \xi^{1}\right)} \frac{\partial}{\partial \xi^{2}}\left[\phi_{\xi}\left(1+\frac{a_{0} \xi^{1}}{c^{2}}\right)^{2}\right]-\frac{1}{\left(1+\frac{a_{0} \xi^{1}}{c^{2}}\right)} \frac{\partial A_{\xi^{2}}}{c \partial \xi^{0}}\right]+\sinh \left(\frac{a_{0}}{c} \xi^{0}\right)\left[\frac{\partial A_{\xi^{2}}}{\partial \xi^{1}}-\frac{\partial A_{\xi^{1}}}{\partial \xi^{2}}\right]
\end{aligned}
$$

The $z$-component of the electric field in the inertial frame can be written as

$$
\begin{aligned}
& E_{z}=-\frac{\partial \phi}{\partial z}-\frac{\partial A_{z}}{c \partial t}=-\frac{\partial}{\partial \xi^{3}}\left[\cosh \left(\frac{a_{0} \xi^{0}}{c}\right)\left(1+\frac{a_{0}}{c^{2}} \xi^{1}\right) \phi_{\xi}+\sinh \left(\frac{a_{0} \xi^{0}}{c}\right) A_{\xi^{1}}\right] \\
& -\left[\frac{\cosh \left(\frac{a_{0} \xi^{0}}{c}\right)}{\left(1+\frac{a_{0} \xi^{1}}{c^{2}}\right)} \frac{\partial}{c \partial \xi^{0}}-\sinh \left(\frac{a_{0} \xi^{0}}{c}\right) \frac{\partial}{\partial \xi^{1}}\right] A_{\xi^{3}} \\
& =-\left(1+\frac{a_{0} \xi^{1}}{c^{2}}\right) \cosh \left(\frac{a_{0} \xi^{0}}{c}\right) \frac{\partial \phi_{\xi}}{\partial \xi^{3}}-\frac{1}{\left(1+\frac{a_{0} \xi^{1}}{c^{2}}\right)} \cosh \left(\frac{a_{0} \xi^{0}}{c}\right) \frac{\partial A_{\xi^{3}}}{c \partial \xi^{0}}+\sinh \left(\frac{a_{0}}{c} \xi^{0}\right)\left[\frac{\partial A_{\xi^{3}}}{\partial \xi^{1}}-\frac{\partial A_{\xi^{1}}}{\partial \xi^{3}}\right] \\
& =\cosh \left(\frac{a_{0}}{c} \xi^{0}\right)\left[-\frac{1}{\left(1+\frac{a_{0}}{c^{2}} \xi^{1}\right)} \frac{\partial}{\partial \xi^{3}}\left[\phi_{\xi}\left(1+\frac{a_{0} \xi^{1}}{c^{2}}\right)^{2}\right]-\frac{1}{\left(1+\frac{a_{0} \xi^{1}}{c^{2}}\right)} \frac{\partial A_{\xi^{3}}}{c \partial \xi^{0}}\right]+\sinh \left(\frac{a_{0}}{c} \xi^{0}\right)\left[\frac{\partial A_{\xi^{3}}}{\partial \xi^{1}}-\frac{\partial A_{\xi^{1}}}{\partial \xi^{3}}\right]
\end{aligned}
$$

Now, for the magnetic field, the $x, y$ and $z$ components are given by Eqns. (23) to (25) as follows:

$$
B_{x}=\frac{\partial A_{z}}{\partial y}-\frac{\partial A_{y}}{\partial z}=\frac{\partial A_{\xi^{3}}}{\partial \xi^{2}}-\frac{\partial A_{\xi^{2}}}{\partial \xi^{3}}
$$

$$
\begin{aligned}
B_{y} & =\frac{\partial A_{x}}{\partial z}-\frac{\partial A_{z}}{\partial x}=\frac{\partial A_{x}}{\partial \xi^{3}}-\frac{\partial A_{\xi^{3}}}{\partial x} \\
& =\frac{\partial}{\partial \xi^{3}}\left[\sinh \left(\frac{a_{0} \xi^{0}}{c}\right)\left(1+\frac{a_{0}}{c^{2}} \xi^{1}\right) \phi_{\xi}+\cosh \left(\frac{a_{0} \xi^{0}}{c}\right) A_{\xi^{1}}\right]-\left[-\frac{\sinh \left(\frac{a_{0} \xi^{0}}{c}\right)}{\left(1+\frac{a_{0} \xi^{1}}{c^{2}}\right)} \frac{\partial}{c \partial \xi^{0}}+\cosh \left(\frac{a_{0} \xi^{0}}{c}\right) \frac{\partial}{\partial \xi^{1}}\right] A_{\xi^{3}}
\end{aligned}
$$




$$
=\cosh \left(\frac{a_{0}}{c} \xi^{0}\right)\left[\frac{\partial A_{\xi^{1}}}{\partial \xi^{3}}-\frac{\partial A_{\xi^{3}}}{\partial \xi^{1}}\right]-\sinh \left(\frac{a_{0}}{c} \xi^{0}\right)\left[-\frac{1}{\left(1+\frac{a_{0}}{c^{2}} \xi^{1}\right)} \frac{\partial}{\partial \xi^{3}}\left[\phi_{\xi}\left(1+\frac{a_{0} \xi^{1}}{c^{2}}\right)^{2}\right]-\frac{1}{\left(1+\frac{a_{0} \xi^{1}}{c^{2}}\right)} \frac{\partial A_{\xi^{3}}}{c \partial \xi^{0}}\right]
$$

$$
\begin{aligned}
B_{z} & =\frac{\partial A_{y}}{\partial x}-\frac{\partial A_{x}}{\partial y}=\frac{\partial A_{\xi^{2}}}{\partial x}-\frac{\partial A_{x}}{\partial \xi^{2}} \\
& =\left[-\frac{\sinh \left(\frac{a_{0} \xi^{0}}{c}\right)}{\left(1+\frac{a_{0} \xi^{1}}{c^{2}}\right)} \frac{\partial}{c \partial \xi^{0}}+\cosh \left(\frac{a_{0} \xi^{0}}{c}\right) \frac{\partial}{\partial \xi^{1}}\right] A_{\xi^{3}}-\frac{\partial}{\partial \xi^{2}}\left[\sinh \left(\frac{a_{0} \xi^{0}}{c}\right)\left(1+\frac{a_{0}}{c^{2}} \xi^{1}\right) \phi_{\xi}+\cosh \left(\frac{a_{0} \xi^{0}}{c}\right) A_{\xi^{1}}\right] \\
& =\cosh \left(\frac{a_{0}}{c} \xi^{0}\right)\left[\frac{\partial A_{\xi^{2}}}{\partial \xi^{1}}-\frac{\partial A_{\xi^{1}}}{\partial \xi^{2}}\right]+\sinh \left(\frac{a_{0}}{c} \xi^{0}\right)\left[-\frac{1}{\left(1+\frac{a_{0}}{c^{2}} \xi^{1}\right)} \frac{\partial}{\partial \xi^{2}}\left[\phi_{\xi}\left(1+\frac{a_{0} \xi^{1}}{c^{2}}\right)^{2}\right]-\frac{1}{\left(1+\frac{a_{0} \xi^{1}}{c^{2}}\right)} \frac{\partial A_{\xi^{2}}}{c \xi^{0}}\right]
\end{aligned}
$$

Hence, we can define the electromagnetic field $\left(\vec{E}_{\xi}, \vec{B}_{\xi}\right)$ in Rindler space-time. This is given as

$$
\vec{E}_{\xi}=-\frac{1}{\left(1+\frac{a_{0} \xi^{1}}{c^{2}}\right)} \vec{\nabla}_{\xi}\left\{\phi_{\xi}\left(1+\frac{a_{0} \xi^{1}}{c^{2}}\right)^{2}\right\}-\frac{1}{\left(1+\frac{a_{0} \xi^{1}}{c^{2}}\right)} \frac{\partial \vec{A}_{\xi}}{c \partial \xi^{0}}
$$$$
\vec{B}_{\xi}=\vec{\nabla}_{\xi} \times \vec{A}_{\xi}
$$

Now,

$$
\vec{\nabla}_{\xi}=\left(\frac{\partial}{\partial \xi^{1}}, \frac{\partial}{\partial \xi^{2}}, \frac{\partial}{\partial \xi^{3}}\right), \vec{A}_{\xi}=\left(A_{\xi^{1}}, A_{\xi^{2}}, A_{\xi^{3}}\right)
$$

We then obtain the transformation of the electromagnetic field as

$$
\begin{aligned}
E_{x} & =-\frac{1}{\left(1+\frac{a_{0} \xi^{1}}{c^{2}}\right)} \frac{\partial}{\partial \xi^{1}}\left\{\phi_{\xi}\left(1+\frac{a_{0} \xi^{1}}{c^{2}}\right)^{2}\right\}-\frac{1}{\left(1+\frac{a_{0} \xi^{1}}{c^{2}}\right)} \frac{\partial A_{\xi^{1}}}{c \xi^{0}} \\
& =E_{\xi^{1}}, \\
E_{y} & =E_{\xi^{2}} \cosh \left(\frac{a_{0} \xi^{0}}{c}\right)+B_{\xi^{3}} \sinh \left(\frac{a_{0} \xi^{0}}{c}\right), \\
E_{z} & =E_{\xi^{3}} \cosh \left(\frac{a_{0} \xi^{0}}{c}\right)-B_{\xi^{2}} \sinh \left(\frac{a_{0} \xi^{0}}{c}\right) \\
B_{x} & =B_{\xi^{1}},
\end{aligned}
$$

$$
\begin{aligned}
& B_{y}=B_{\xi^{2}} \cosh \left(\frac{a_{0} \xi^{0}}{c}\right)-E_{\xi^{3}} \sinh \left(\frac{a_{0} \xi^{0}}{c}\right) \\
& B_{z}=B_{\xi^{3}} \cosh \left(\frac{a_{0} \xi^{0}}{c}\right)+E_{\xi^{2}} \sinh \left(\frac{a_{0} \xi^{0}}{c}\right)
\end{aligned}
$$

Hence, we can find the matrix of the transformation of the electro-magnetic field.

$$
\begin{gathered}
E_{x}=E_{\xi^{1}}, B_{x}=B_{\xi^{1}}, \\
\left(\begin{array}{l}
E_{y} \\
B_{y} \\
E_{z} \\
B_{z}
\end{array}\right)=H\left(\begin{array}{c}
E_{\xi^{2}} \\
B_{\xi^{2}} \\
E_{\xi^{3}} \\
B_{\xi^{3}}
\end{array}\right) \\
H=\left(\begin{array}{cccc}
\cosh \left(\frac{a_{0} \xi^{0}}{c}\right) & 0 & 0 & \sinh \left(\frac{a_{0} \xi^{0}}{c}\right) \\
0 & \cosh \left(\frac{a_{0} \xi^{0}}{c}\right) & -\sinh \left(\frac{a_{0} \xi^{0}}{c}\right) & 0 \\
0 & -\sinh \left(\frac{a_{0} \xi^{0}}{c}\right) & \cosh \left(\frac{a_{0} \xi^{0}}{c}\right) & 0 \\
\sinh \left(\frac{a_{0} \xi^{0}}{c}\right) & 0 & 0 & \cosh \left(\frac{a_{0} \xi^{0}}{c}\right)
\end{array}\right)
\end{gathered}
$$

Similarly, we can obtain the matrix of the inversetransformation of the electromagnetic field. 


$$
\begin{aligned}
& E_{\xi^{1}}=E_{x}, B_{\xi^{1}}=B_{x} \\
& \left(\begin{array}{c}
E_{\xi^{2}} \\
B_{\xi^{2}} \\
E_{\xi^{3}} \\
B_{\xi^{3}}
\end{array}\right)=H^{-1}\left(\begin{array}{l}
E_{y} \\
B_{y} \\
E_{z} \\
B_{z}
\end{array}\right) \\
& E_{\xi^{1}}=E_{x}, B_{\xi^{1}}=B_{x} \\
& E_{\xi^{2}}=E_{y} \cosh \left(\frac{a_{0} \xi^{0}}{c}\right)-B_{z} \sinh \left(\frac{a_{0} \xi^{0}}{c}\right), \\
& B_{\xi^{2}}=B_{y} \cosh \left(\frac{a_{0} \xi^{0}}{c}\right)+E_{z} \sinh \left(\frac{a_{0} \xi^{0}}{c}\right) \\
& E_{\xi^{3}}=E_{z} \cosh \left(\frac{a_{0} \xi^{0}}{c}\right)+B_{y} \sinh \left(\frac{a_{0} \xi^{0}}{c}\right) \\
& B_{\xi^{3}}=B_{z} \cosh \left(\frac{a_{0} \xi^{0}}{c}\right)-E_{y} \sinh \left(\frac{a_{0} \xi^{0}}{c}\right)
\end{aligned}
$$$$
H^{-1}=\left(\begin{array}{cccc}
\cosh \left(\frac{a_{0} \xi^{0}}{c}\right) & 0 & 0 & -\sinh \left(\frac{a_{0} \xi^{0}}{c}\right) \\
0 & \cosh \left(\frac{a_{0} \xi^{0}}{c}\right) & \sinh \left(\frac{a_{0} \xi^{0}}{c}\right) & 0 \\
0 & \sinh \left(\frac{a_{0} \xi^{0}}{c}\right) & \cosh \left(\frac{a_{0} \xi^{0}}{c}\right) & 0 \\
-\sinh \left(\frac{a_{0} \xi^{0}}{c}\right) & 0 & 0 & \cosh \left(\frac{a_{0} \xi^{0}}{c}\right)
\end{array}\right)
$$

(see also Ref. [2]). Hence, the inverse-

If we apply Lorentz gauge transformation, Eqn. (10) to electromagnetic field, Eqn. (26) in Rindler space-time, then we get

$$
\phi_{\xi} \rightarrow \phi_{\xi}-\frac{1}{c} \frac{\partial \Lambda}{\partial \xi^{0}} \frac{1}{\left(1+\frac{a_{0} \xi^{1}}{c^{2}}\right)^{2}}, \quad \vec{A}_{\xi} \rightarrow \vec{A}_{\xi}+\vec{\nabla}_{\xi} \Lambda
$$

Where, $\Lambda$ is a scalar function.

$$
\begin{gathered}
\vec{E}_{\xi}=-\frac{1}{\left(1+\frac{a_{0} \xi^{1}}{c^{2}}\right)} \vec{\nabla}_{\xi}\left\{\phi_{\xi}\left(1+\frac{a_{0} \xi^{1}}{c^{2}}\right)^{2}\right\}+\frac{1}{\left(1+\frac{a_{0} \xi^{1}}{c^{2}}\right)} \vec{\nabla}_{\xi} \frac{\partial \Lambda}{c \partial \xi^{0}} \\
-\frac{1}{\left(1+\frac{a_{0} \xi^{1}}{c^{2}}\right)} \frac{\partial \vec{A}_{\xi}}{c \partial \xi^{0}}-\frac{1}{\left(1+\frac{a_{0} \xi^{1}}{c^{2}}\right)} \frac{\partial}{c \partial \xi^{0}} \vec{\nabla}_{\xi} \Lambda \\
=-\frac{1}{\left(1+\frac{a_{0} \xi^{1}}{c^{2}}\right)} \vec{\nabla}_{\xi}\left\{\phi_{\xi}\left(1+\frac{a_{0} \xi^{1}}{c^{2}}\right)^{2}\right\}-\frac{1}{\left(1+\frac{a_{0} \xi^{1}}{c^{2}}\right)} \frac{\partial \vec{A}_{\xi}}{c \partial \xi^{0}}
\end{gathered}
$$

$$
\vec{B}_{\xi}=\vec{\nabla}_{\xi} \times \vec{A}_{\xi}+\vec{\nabla}_{\xi} \times \vec{\nabla}_{\xi} \Lambda=\vec{\nabla}_{\xi} \times \vec{A}_{\xi}
$$

If we apply Lorentz gauge transformation, Eqn. (10) to the transformation of the electromagnetic 4vector potential, and Eqn. (13), then we can obtain the following results.

$$
\begin{aligned}
\phi-\frac{1}{c} \frac{\partial \Lambda}{\partial t}= & \cosh \left(\frac{a_{0} \xi^{0}}{c}\right)\left(1+\frac{a_{0}}{c^{2}} \xi^{1}\right)\left\{\phi_{\xi}-\frac{1}{c} \frac{\partial \Lambda}{\partial \xi^{0}} \frac{1}{\left(1+\frac{a_{0} \xi^{1}}{c^{2}}\right)^{2}}\right\} \\
& +\sinh \left(\frac{a_{0} \xi^{0}}{c}\right)\left(A_{\xi^{1}}+\frac{\partial \Lambda}{\partial \xi^{1}}\right) \\
A_{x}+\frac{\partial \Lambda}{\partial x}= & \sinh \left(\frac{a_{0} \xi^{0}}{c}\right)\left(1+\frac{a_{0}}{c^{2}} \xi^{1}\right)\left\{\phi_{\xi}-\frac{1}{c} \frac{\partial \Lambda}{\partial \xi^{0}} \frac{1}{\left(1+\frac{a_{0} \xi^{1}}{c^{2}}\right)^{2}}\right\} \\
& +\cosh \left(\frac{a_{0} \xi^{0}}{c}\right)\left(A_{\xi^{1}}+\frac{\partial \Lambda}{\partial \xi^{1}}\right) \\
A_{y}+\frac{\partial \Lambda}{\partial y}= & A_{\xi^{2}}+\frac{\partial \Lambda}{\partial \xi^{2}}, A_{z}+\frac{\partial \Lambda}{\partial z}=A_{\xi^{3}}+\frac{\partial \Lambda}{\partial \xi^{3}}
\end{aligned}
$$

\section{Electromagnetic Field Equation in the} Rindler Space-time

Maxwell equation is

$$
\begin{aligned}
& \vec{\nabla} \cdot \vec{E}=4 \pi \rho \\
& \vec{\nabla} \times \vec{B}=\frac{\partial \vec{E}}{c \partial t}+\frac{4 \pi}{c} \vec{j} \\
& \vec{\nabla} \cdot \vec{B}=0 \\
& \vec{\nabla} \times \vec{E}=-\frac{\partial \vec{B}}{c \partial t}
\end{aligned}
$$


We shall now perform computation to derive Maxwell equations in Rindler space-time. For this purpose, we shall compute it by using the electromagnetic field transformation, Eqn. (27) and the transformation of differential operation as given in Eqn. (17).

Let us first deal with the transformation of 4vector, the charge density and the electrical current density $(c \rho, \vec{j})=\rho_{0} \frac{d x^{\alpha}}{d \tau}$ and $\left(c \rho_{\xi}, \vec{j}_{\xi}\right)$

Where,

$$
\begin{aligned}
& \rho=\rho_{\xi}\left(1+\frac{a_{0} \xi^{1}}{c^{2}}\right) \cosh \left(\frac{a_{0} \xi^{0}}{c}\right)+\frac{j_{\xi^{1}}}{c} \sinh \left(\frac{a_{0} \xi^{0}}{c}\right) \\
& j_{x}=j_{\xi^{1}} \cosh \left(\frac{a_{0} \xi^{0}}{c}\right)+c \rho_{\xi}\left(1+\frac{a_{0}}{c^{2}} \xi^{1}\right) \sinh \left(\frac{a_{0} \xi^{0}}{c}\right), \\
& j_{y}=j_{\xi^{2}}, j_{z}=j_{\xi^{3}}
\end{aligned}
$$

Now, 4-vector $\left(c \rho_{\xi}, \vec{j}_{\xi}\right)=\rho_{0} \frac{d \xi^{\alpha}}{d \tau}$ is defined in Rindler space-time.

The first of the Maxwell equations is given in the inertial frame as follows:

$$
\begin{aligned}
& \text { 1. } \vec{\nabla} \cdot \vec{E}=4 \pi \rho \\
& E_{x}=E_{\xi 1} \text {, } \\
& E_{y}=E_{\xi^{2}} \cosh \left(\frac{a_{0} \xi^{0}}{c}\right)+B_{\xi^{3}} \sinh \left(\frac{a_{0} \xi^{0}}{c}\right), \\
& E_{z}=E_{\xi^{3}} \cosh \left(\frac{a_{0} \xi^{0}}{c}\right)-B_{\xi^{2}} \sinh \left(\frac{a_{0} \xi^{0}}{c}\right) \\
& 4 \pi \rho=\frac{\partial E_{x}}{\partial x}+\frac{\partial E_{y}}{\partial y}+\frac{\partial E_{z}}{\partial z} \\
& =\left[-\frac{\sinh \left(\frac{a_{0} \xi^{0}}{c}\right)}{\left(1+\frac{a_{0} \xi^{1}}{c^{2}}\right)} \frac{\partial}{c \partial \xi^{0}}+\cosh \left(\frac{a_{0} \xi^{0}}{c}\right) \frac{\partial}{\partial \xi^{1}}\right] E_{\xi^{1}} \\
& +\frac{\partial}{\partial \xi^{2}}\left[E_{\xi^{2}} \cosh \left(\frac{a_{0} \xi^{0}}{c}\right)+B_{\xi^{3}} \sinh \left(\frac{a_{0} \xi^{0}}{c}\right)\right] \\
& +\frac{\partial}{\partial \xi^{3}}\left[E_{\xi^{3}} \cosh \left(\frac{a_{0} \xi^{0}}{c}\right)-B_{\xi^{2}} \sinh \left(\frac{a_{0} \xi^{0}}{c}\right)\right] \\
& =\cosh \left(\frac{a_{0}}{c} \xi^{0}\right)\left(\vec{\nabla}_{\xi} \cdot \vec{E}_{\xi}\right) \\
& +\sinh \left(\frac{a_{0}}{c} \xi^{0}\right)\left[\frac{\partial B_{\xi^{3}}}{\partial \xi^{2}}-\frac{\partial B_{\xi^{2}}}{\partial \xi^{3}}-\frac{1}{\left(1+\frac{a_{0} \xi^{1}}{c^{2}}\right)} \frac{\partial E_{\xi^{1}}}{c \partial \xi^{0}}\right]
\end{aligned}
$$

2. $\vec{\nabla} \times \vec{B}=\frac{\partial \vec{E}}{c \partial t}+\frac{4 \pi}{c} \vec{j}$

$$
\begin{aligned}
& B_{x}=B_{\xi^{1}} \\
& B_{y}=B_{\xi^{2}} \cosh \left(\frac{a_{0} \xi^{0}}{c}\right)-E_{\xi^{3}} \sinh \left(\frac{a_{0} \xi^{0}}{c}\right) \\
& B_{z}=B_{\xi^{3}} \cosh \left(\frac{a_{0} \xi^{0}}{c}\right)+E_{\xi^{2}} \sinh \left(\frac{a_{0} \xi^{0}}{c}\right)
\end{aligned}
$$

The $\mathrm{x}$-component is:

$$
\begin{aligned}
& \frac{\partial B_{z}}{\partial y}-\frac{\partial B_{y}}{\partial z} \\
= & \frac{\partial}{\partial \xi^{2}}\left[B_{\xi^{3}} \cosh \left(\frac{a_{0} \xi^{0}}{c}\right)+E_{\xi^{2}} \sinh \left(\frac{a_{0} \xi^{0}}{c}\right)\right] \\
& -\frac{\partial}{\partial \xi^{3}}\left[B_{\xi^{2}} \cosh \left(\frac{a_{0} \xi^{0}}{c}\right)-E_{\xi^{3}} \sinh \left(\frac{a_{0} \xi^{0}}{c}\right)\right] \\
= & \cosh \left(\frac{a_{0}}{c} \xi^{0}\right)\left[\frac{\partial B^{3}}{\partial \xi^{2}}-\frac{\partial B_{\xi^{2}}}{\partial \xi^{3}}\right] \\
& +\sinh \left(\frac{a_{0} \xi^{0}}{c}\right)\left[\frac{\partial E_{\xi^{2}}}{\partial \xi^{2}}+\frac{\partial E_{\xi^{3}}}{\partial \xi^{3}}\right] \\
= & \frac{\partial E_{x}}{c \partial t}+\frac{4 \pi}{c} j_{x} \\
& \cosh \left(\frac{a_{0} \xi^{0}}{c}\right) \\
= & \left(1+\frac{a_{0} \xi^{1}}{c^{2}}\right)
\end{aligned}
$$

Hence,

$$
\begin{aligned}
& \frac{4 \pi}{c} j_{x}=\sinh \left(\frac{a_{0} \xi^{0}}{c}\right)\left(\vec{\nabla}_{\xi} \cdot \vec{E}_{\xi}\right) \\
& \quad+\cosh \left(\frac{a_{0} \xi^{0}}{c}\right)\left[\frac{\partial B_{\xi^{3}}}{\partial \xi^{2}}-\frac{\partial B_{\xi^{2}}}{\partial \xi^{3}}-\frac{1}{\left(1+\frac{a_{0} \xi^{1}}{c^{2}}\right)} \frac{\partial E_{\xi^{1}}}{c \partial \xi^{0}}\right]
\end{aligned}
$$

The $y$-component is:

$$
\begin{aligned}
& \frac{\partial B_{x}}{\partial z}-\frac{\partial B_{z}}{\partial x}=\frac{\partial B_{\xi^{1}}}{\partial \xi^{3}} \\
& -\left[-\frac{\sinh \left(\frac{a_{0} \xi^{0}}{c}\right)}{\left(1+\frac{a_{0} \xi^{1}}{c^{2}}\right)} \frac{\partial}{c \partial \xi^{0}}+\cosh \left(\frac{a_{0} \xi^{0}}{c}\right) \frac{\partial}{\partial \xi^{1}}\right]
\end{aligned}
$$




$$
\begin{aligned}
& \cdot\left[B_{\xi^{3}} \cosh \left(\frac{a_{0} \xi^{0}}{c}\right)+E_{\xi^{2}} \sinh \left(\frac{a_{0} \xi^{0}}{c}\right)\right] \\
& =\frac{\partial E_{y}}{c \partial t}+\frac{4 \pi}{c} j_{y} \\
& =\left[\frac{\cosh \left(\frac{a_{0} \xi^{0}}{c}\right)}{\left(1+\frac{a_{0} \xi^{1}}{c^{2}}\right)} \frac{\partial}{c \partial \xi^{0}}-\sinh \left(\frac{a_{0} \xi^{0}}{c}\right) \frac{\partial}{\partial \xi^{1}}\right] \\
& \cdot\left[E_{\xi^{2}} \cosh \left(\frac{a_{0} \xi^{0}}{c}\right)+B_{\xi^{3}} \sinh \left(\frac{a_{0} \xi^{0}}{c}\right)\right]+\frac{4 \pi}{c} j_{y} \\
& \frac{4 \pi}{c} j_{y}=\frac{\partial B_{\xi^{1}}}{\partial \xi^{3}}-\frac{\partial B_{\xi^{3}}}{\partial \xi^{1}}-\frac{1}{\left(1+\frac{a_{0}}{c^{2}} \xi^{1}\right)} \frac{a_{0}}{c^{2}} B_{\xi^{3}} \\
& -\frac{1}{\left(1+\frac{a_{0}}{c^{2}} \xi^{1}\right)} \frac{\partial E_{\xi^{2}}}{c \partial \xi^{0}} \\
& =\frac{1}{\left(1+\frac{a_{0}}{c^{2}} \xi^{1}\right)} \frac{\partial}{\partial \xi^{3}}\left\{B_{\xi^{1}}\left(1+\frac{a_{0}}{c^{2}} \xi^{1}\right)\right\} \\
& -\frac{1}{\left(1+\frac{a_{0}}{c^{2}} \xi^{1}\right)} \frac{\partial}{\partial \xi^{1}}\left\{B_{\xi^{3}}\left(1+\frac{a_{0} \xi^{1}}{c^{2}}\right)\right\}-\frac{1}{\left(1+\frac{a_{0}}{c^{2}} \xi^{1}\right)} \frac{\partial E_{\xi^{2}}}{c \partial \xi^{0}}
\end{aligned}
$$

And, the $z$-component is given as:

$$
\begin{aligned}
& \frac{\partial B_{y}}{\partial x}-\frac{\partial B_{x}}{\partial y} \\
& =\left[-\frac{\sinh \left(\frac{a_{0} \xi^{0}}{c}\right)}{\left(1+\frac{a_{0} \xi^{1}}{c^{2}}\right)} \frac{\partial}{c \partial \xi^{0}}+\cosh \left(\frac{a_{0} \xi^{0}}{c}\right) \frac{\partial}{\partial \xi^{1}}\right] \\
& \cdot\left[B_{\xi^{2}} \cosh \left(\frac{a_{0} \xi^{0}}{c}\right)-E_{\xi^{3}} \sinh \left(\frac{a_{0} \xi^{0}}{c}\right)\right]-\frac{\partial B_{\xi^{1}}}{\partial \xi^{2}} \\
& =\frac{\partial E_{z}}{c \partial t}+\frac{4 \pi}{c} j_{z} \\
& =\left[\frac{\cosh \left(\frac{a_{0} \xi^{0}}{c}\right)}{\left(1+\frac{a_{0} \xi^{1}}{c^{2}}\right)} \frac{\partial}{c \partial \xi^{0}}-\sinh \left(\frac{a_{0} \xi^{0}}{c}\right) \frac{\partial}{\partial \xi^{1}}\right] \\
& \cdot\left[E_{\xi^{3}} \cosh \left(\frac{a_{0} \xi^{0}}{c}\right)-B_{\xi^{2}} \sinh \left(\frac{a_{0} \xi^{0}}{c}\right)\right]+\frac{4 \pi}{c} j_{z}
\end{aligned}
$$

Therefore,

$$
\begin{gathered}
\frac{4 \pi}{c} j_{z}=\frac{\partial B_{\xi^{2}}}{\partial \xi^{1}}-\frac{\partial B_{\xi^{1}}}{\partial \xi^{2}}+\frac{1}{\left(1+\frac{a_{0}}{c^{2}} \xi^{1}\right)} \frac{a_{0}}{c^{2}} B_{\xi^{2}} \\
-\frac{1}{\left(1+\frac{a_{0}}{c^{2}} \xi^{1}\right)} \frac{\partial E_{\xi^{3}}}{c \partial \xi^{0}} \\
=\frac{1}{\left(1+\frac{a_{0}}{c^{2}} \xi^{1}\right)} \frac{\partial}{\partial \xi^{1}}\left\{B_{\xi^{2}}\left(1+\frac{a_{0}}{c^{2}} \xi^{1}\right)\right\} \\
-\frac{1}{\left(1+\frac{a_{0}}{c^{2}} \xi^{1}\right)} \frac{\partial}{\partial \xi^{2}}\left\{B_{\xi^{1}}\left(1+\frac{a_{0} \xi^{1}}{c^{2}}\right)\right\}-\frac{1}{\left(1+\frac{a_{0}}{c^{2}} \xi^{1}\right)} \frac{\partial E_{\xi^{3}}}{c \partial \xi^{0}}
\end{gathered}
$$

The third law described by Maxwell equations in inertial frame is:

3. $\vec{\nabla} \cdot \vec{B}=0$

$$
\begin{gathered}
\vec{\nabla} \cdot \vec{B}=\frac{\partial B_{x}}{\partial x}+\frac{\partial B_{y}}{\partial y}+\frac{\partial B_{z}}{\partial z} \\
=\left[-\frac{\sinh \left(\frac{a_{0} \xi^{0}}{c}\right)}{\left(1+\frac{a_{0} \xi^{1}}{c^{2}}\right)} \frac{\partial}{c \partial \xi^{0}}+\cosh \left(\frac{a_{0} \xi^{0}}{c}\right) \frac{\partial}{\partial \xi^{1}}\right] B_{\xi^{1}} \\
+\frac{\partial}{\partial \xi^{2}}\left[B_{\xi^{2}} \cosh \left(\frac{a_{0} \xi^{0}}{c}\right)-E_{\xi^{3}} \sinh \left(\frac{a_{0} \xi^{0}}{c}\right)\right] \\
+\frac{\partial}{\partial \xi^{3}}\left[B_{\xi^{3}} \cosh \left(\frac{a_{0} \xi^{0}}{c}\right)+E_{\xi^{2}} \sinh \left(\frac{a_{0} \xi^{0}}{c}\right)\right] \\
=\cosh \left(\frac{a_{0} \xi^{0}}{c}\right)\left(\vec{\nabla}_{\xi} \cdot \vec{B}_{\xi}\right)+\sinh \left(\frac{a_{0} \xi^{0}}{c}\right)\left[-\left(-\frac{\partial E_{\xi^{2}}}{\partial \xi^{3}}+\frac{\partial E_{\xi^{3}}}{\partial \xi^{2}}\right)\right. \\
\left.\quad \frac{1}{\left(1+\frac{a_{0}}{c^{2}} \xi^{1}\right)} \frac{\partial B_{\xi^{1}}}{c \partial \xi^{0}}\right]=0
\end{gathered}
$$

Now, the fourth equation of Maxwell in the inertial frame is given as:

4. $\vec{\nabla} \times \vec{E}=-\frac{\partial \vec{B}}{c \partial t}$

$$
\begin{aligned}
& E_{x}=E_{\xi^{1}}, \\
& E_{y}=E_{\xi^{2}} \cosh \left(\frac{a_{0} \xi^{0}}{c}\right)+B_{\xi^{3}} \sinh \left(\frac{a_{0} \xi^{0}}{c}\right), \\
& E_{z}=E_{\xi^{3}} \cosh \left(\frac{a_{0} \xi^{0}}{c}\right)-B_{\xi^{2}} \sinh \left(\frac{a_{0} \xi^{0}}{c}\right)
\end{aligned}
$$


The $x$-component is given as:

$$
\begin{aligned}
& \frac{\partial E_{z}}{\partial y}-\frac{\partial E_{y}}{\partial z} \\
& =\frac{\partial}{\partial \xi^{2}}\left[E_{\xi^{3}} \cosh \left(\frac{a_{0} \xi^{0}}{c}\right)-B_{\xi^{2}} \sinh \left(\frac{a_{0} \xi^{0}}{c}\right)\right]-\frac{\partial}{\partial \xi^{3}} \\
& {\left[E_{\xi^{2}} \cosh \left(\frac{a_{0} \xi^{0}}{c}\right)+B_{\xi^{3}} \sinh \left(\frac{a_{0} \xi^{0}}{c}\right)\right]} \\
& =\cosh \left(\frac{a_{0}}{c} \xi^{0}\right)\left[\frac{\partial E_{\xi^{3}}}{\partial \xi^{2}}-\frac{\partial E_{\xi^{2}}}{\partial \xi^{3}}\right] \\
& =-\frac{\partial B_{x}}{c \partial t} \quad \sinh \left(\frac{a_{0} \xi^{0}}{c}\right)\left[\frac{\partial B^{2}}{\partial \xi^{2}}+\frac{\partial B_{\xi^{3}}}{\partial \xi^{3}}\right] \\
& =-\left[\frac{\cosh \left(\frac{a_{0} \xi^{0}}{c}\right)}{\left(1+\frac{a_{0} \xi^{1}}{c^{2}}\right)} \frac{\partial}{c \partial \xi^{0}}-\sinh \left(\frac{a_{0} \xi^{0}}{c}\right) \frac{\partial}{\partial \xi^{1}}\right] B_{\xi^{1}}
\end{aligned}
$$

Hence,

$$
\begin{aligned}
& -\sinh \left(\frac{a_{0} \xi^{0}}{c}\right)\left(\vec{\nabla}_{\xi} \cdot \vec{B}_{\xi}\right) \\
& +\cosh \left(\frac{a_{0} \xi^{0}}{c}\right)\left[\left(\frac{\partial E_{\xi^{3}}}{\partial \xi^{2}}-\frac{\partial E_{\xi^{2}}}{\partial \xi^{3}}\right)+\frac{1}{\left(1+\frac{a_{0} \xi^{1}}{c^{2}}\right)} \frac{\partial B_{\xi^{1}}}{c \partial \xi^{0}}\right]=0
\end{aligned}
$$

The $y$-component is:

$$
\begin{aligned}
& \frac{\partial E_{x}}{\partial z}-\frac{\partial E_{z}}{\partial x}=\frac{\partial E_{\xi^{1}}}{\partial \xi^{3}} \\
& -\left[-\frac{\sinh \left(\frac{a_{0} \xi^{0}}{c}\right)}{\left(1+\frac{a_{0} \xi^{1}}{c^{2}}\right)} \frac{\partial}{c \partial \xi^{0}}+\cosh \left(\frac{a_{0} \xi^{0}}{c}\right) \frac{\partial}{\partial \xi^{1}}\right] \\
& \cdot\left[E_{\xi^{3}} \cosh \left(\frac{a_{0} \xi^{0}}{c}\right)-B_{\xi^{2}} \sinh \left(\frac{a_{0} \xi^{0}}{c}\right)\right] \\
& =-\frac{\partial B_{y}}{c \partial t} \\
& =-\left[\frac{\cosh \left(\frac{a_{0} \xi^{0}}{c}\right)}{\left(1+\frac{a_{0} \xi^{1}}{c^{2}}\right)} \frac{\partial}{c \partial \xi^{0}}-\sinh \left(\frac{a_{0} \xi^{0}}{c}\right) \frac{\partial}{\partial \xi^{1}}\right] \\
& \cdot\left[B_{\xi^{2}} \cosh \left(\frac{a_{0} \xi^{0}}{c}\right)-E_{\xi^{3}} \sinh \left(\frac{a_{0} \xi^{0}}{c}\right)\right]
\end{aligned}
$$

$$
\begin{aligned}
& \frac{\partial E_{\xi^{1}}}{\partial \xi^{3}}-\frac{\partial E_{\xi^{3}}}{\partial \xi^{1}}-\frac{1}{\left(1+\frac{a_{0}}{c^{2}} \xi^{1}\right)} \frac{a_{0}}{c^{2}} E_{\xi^{3}}+\frac{1}{\left(1+\frac{a_{0}}{c^{2}} \xi^{1}\right)} \frac{\partial B_{\xi^{2}}}{c \xi^{0}} \\
& =\frac{1}{\left(1+\frac{a_{0}}{c^{2}} \xi^{1}\right)} \frac{\partial}{\partial \xi^{3}}\left\{E_{\xi^{1}}\left(1+\frac{a_{0}}{c^{2}} \xi^{1}\right)\right\} \\
& -\frac{1}{\left(1+\frac{a_{0}}{c^{2}} \xi^{1}\right)} \frac{\partial}{\partial \xi^{1}}\left\{E_{\xi^{3}}\left(1+\frac{a_{0} \xi^{1}}{c^{2}}\right)\right\}+\frac{1}{\left(1+\frac{a_{0}}{c^{2}} \xi^{1}\right)} \frac{\partial B_{\xi^{2}}}{c \partial \xi^{0}} \\
& =0
\end{aligned}
$$

The $z$-component is given as:

$$
\begin{aligned}
& \frac{\partial E_{y}}{\partial x}-\frac{\partial E_{x}}{\partial y} \\
& =\left[-\frac{\sinh \left(\frac{a_{0} \xi^{0}}{c}\right)}{\left(1+\frac{a_{0} \xi^{1}}{c^{2}}\right)} \frac{\partial}{c \partial \xi^{0}}+\cosh \left(\frac{a_{0} \xi^{0}}{c}\right) \frac{\partial}{\partial \xi^{1}}\right]
\end{aligned}
$$$$
\cdot\left[E_{\xi^{2}} \cosh \left(\frac{a_{0} \xi^{0}}{c}\right)+B_{\xi^{3}} \sinh \left(\frac{a_{0} \xi^{0}}{c}\right)\right]-\frac{\partial E_{\xi^{1}}}{\partial \xi^{2}}
$$$$
=-\frac{\partial B_{z}}{c \partial t}
$$$$
=-\left[\frac{\cosh \left(\frac{a_{0} \xi^{0}}{c}\right)}{\left(1+\frac{a_{0} \xi^{1}}{c^{2}}\right)} \frac{\partial}{c \partial \xi^{0}}-\sinh \left(\frac{a_{0} \xi^{0}}{c}\right) \frac{\partial}{\partial \xi^{1}}\right]
$$

$$
\begin{aligned}
\cdot & {\left[B_{\xi^{3}} \cosh \left(\frac{a_{0} \xi^{0}}{c}\right)+E_{\xi^{2}} \sinh \left(\frac{a_{0} \xi^{0}}{c}\right)\right] } \\
& \frac{\partial E_{\xi^{2}}}{\partial \xi^{1}}-\frac{\partial E_{\xi^{1}}}{\partial \xi^{2}}+\frac{1}{\left(1+\frac{a_{0}}{c^{2}} \xi^{1}\right)} \frac{a_{0}}{c^{2}} E_{\xi^{2}}+\frac{1}{\left(1+\frac{a_{0}}{c^{2}} \xi^{1}\right)} \frac{\partial B_{\xi^{3}}}{c \xi^{0}} \\
= & \frac{1}{\left(1+\frac{a_{0}}{c^{2}} \xi^{1}\right)} \frac{\partial}{\partial \xi^{1}}\left\{E_{\xi^{2}}\left(1+\frac{a_{0}}{c^{2}} \xi^{1}\right)\right\} \\
- & \frac{1}{\left(1+\frac{a_{0}}{c^{2}} \xi^{1}\right)} \frac{\partial}{\partial \xi^{2}}\left\{E_{\xi^{1}}\left(1+\frac{a_{0} \xi^{1}}{c^{2}}\right)\right\}+\frac{1}{\left(1+\frac{a_{0}}{c^{2}} \xi^{1}\right)} \frac{\partial B_{\xi^{3}}}{c \partial \xi^{0}} \\
= & 0
\end{aligned}
$$

Therefore, we obtain the electromagnetic field equation from Eqns. (35)-(42) in Rindler spacetime (see also Ref. [1]), which is given as

$$
\vec{\nabla}_{\xi} \cdot \vec{E}_{\xi}=4 \pi \rho_{\xi}\left(1+\frac{a_{0} \xi^{1}}{c^{2}}\right)
$$




$$
\begin{gathered}
\frac{1}{\left(1+\frac{a_{0} \xi^{1}}{c^{2}}\right)} \vec{\nabla}_{\xi} \times\left\{\vec{B}_{\xi}\left(1+\frac{a_{0} \xi^{1}}{c^{2}}\right)\right\} \\
=\frac{1}{\left(1+\frac{a_{0} \xi^{1}}{c^{2}}\right)} \frac{\partial \vec{E}_{\xi}}{c \partial \xi^{0}}+\frac{4 \pi}{c} \vec{j}_{\xi} \\
\frac{1}{\left(1+\frac{a_{0} \xi^{1}}{c^{2}}\right)} \vec{\nabla}_{\xi} \times\left\{\vec{E}_{\xi}\left(1+\frac{a_{0} \xi^{1}}{c^{2}}\right)\right\}=-\frac{1}{\left(1+\frac{a_{0} \xi^{1}}{c^{2}}\right)} \frac{\partial \vec{B}_{\xi}}{c \xi^{0}} \\
\vec{E}_{\xi}=\left(E_{\xi^{1}}, E_{\xi^{2}}, E_{\xi^{3}}\right), \vec{B}_{\xi}=\left(B_{\xi^{1}}, B_{\xi^{2}}, B_{\xi^{3}}\right), \\
\vec{\nabla}_{\xi}=\left(\frac{\partial}{\partial \xi^{1}}, \frac{\partial}{\partial \xi^{2}}, \frac{\partial}{\partial \xi^{3}}\right)
\end{gathered}
$$

We know that del Castillio and Sanchez [1] already discovered Maxwell equations in a uniformly accelerated frame in vacuum.

Hence, the transformation of 4-vector $(c \rho, \vec{j})=\rho_{0} \frac{d x^{\alpha}}{d \tau}$ is

$$
\begin{aligned}
& \rho=\rho_{\xi}\left(1+\frac{a_{0} \xi^{1}}{c^{2}}\right) \cosh \left(\frac{a_{0} \xi^{0}}{c}\right)+\frac{j_{\xi^{1}}}{c} \sinh \left(\frac{a_{0} \xi^{0}}{c}\right) \\
& j_{x}=j_{\xi^{1}} \cosh \left(\frac{a_{0} \xi^{0}}{c}\right)+c \rho_{\xi}\left(1+\frac{a_{0}}{c^{2}} \xi^{1}\right) \sinh \left(\frac{a_{0} \xi^{0}}{c}\right) \\
& j_{y}=j_{\xi^{2}}, j_{z}=j_{\xi^{3}}
\end{aligned}
$$

Now, 4-vector $\left(c \rho_{\xi}, \vec{j}_{\xi}\right)=\rho_{0} \frac{d \xi^{\alpha}}{d \tau}$.

For instance, we know that the spherical charge density $\rho_{\xi}$ of a stationary accelerated frame in a charged huge sphere is

$$
\begin{gathered}
t=\xi^{0}=0,0 \leq x=\xi^{1}, y=\xi^{2}, z=\xi^{3} \leq R, \\
\vec{E}=\vec{E}_{\xi}=\frac{Q}{R^{3}} \vec{r}, \quad \rho=\rho_{0}=\frac{Q}{V}=\frac{3 Q}{4 \pi R^{3}}, \\
\vec{j}=\vec{j}_{\xi}=\overrightarrow{0}
\end{gathered}
$$

Generally, the continuity equation (i.e., conservation laws for the charge density and the electrical current) in Rindler space-time is given by

$$
\begin{aligned}
& 0=j_{; \mu}^{\mu}=\frac{\partial j^{\mu}}{\partial \xi^{\mu}}+\Gamma_{\mu \rho}^{\mu} j^{\rho}, \\
& \Gamma_{\mu \rho}^{\mu}=\Gamma_{01}^{0}=\frac{1}{2} g^{00}\left(\frac{\partial g_{00}}{\partial \xi^{1}}\right)=\frac{a_{0}}{c^{2}} \frac{1}{\left(1+\frac{a_{0}}{c^{2}} \xi^{1}\right)} \\
& g^{00}=-\frac{1}{\left(1+\frac{a_{0} \xi^{1}}{c^{2}}\right)^{2}}, g^{11}=g^{22}=g^{33}=1 \\
& 0=\frac{\partial \rho}{\partial t}+\vec{\nabla} \cdot \vec{j}=\frac{\partial \rho_{\xi}}{\partial \xi^{0}}+\vec{\nabla}_{\xi} \cdot \vec{j}_{\xi}+\frac{j_{\xi^{1}} a_{0}}{c^{2}} \frac{1}{\left(1+\frac{a_{0} \xi^{1}}{c^{2}}\right)}
\end{aligned}
$$

We now treat the Lorentz gauge transformation by using Eqn. (10) for the electromagnetic field equations and Eqns. (44a) to (44d) in Rindler space-time. The computation is straightforward by using the Lorentz gauge transformation, Eqn. (10) and the Lorentz gauge fixing condition, Eqn. (12). Eqn. (44a) is

$$
\begin{aligned}
\vec{\nabla}_{\xi} \cdot \vec{E}_{\xi} & =\vec{\nabla}_{\xi} \cdot\left\{-\frac{1}{\left(1+\frac{a_{0} \xi^{1}}{c^{2}}\right)} \vec{\nabla}_{\xi}\left\{\phi_{\xi}\left(1+\frac{a_{0} \xi^{1}}{c^{2}}\right)^{2}\right\}-\frac{1}{\left(1+\frac{a_{0} \xi^{1}}{c^{2}}\right)} \frac{\partial \vec{A}_{\xi}}{c \partial \xi^{0}}\right\} \\
& =-\vec{\nabla}_{\xi}\left\{\frac{1}{\left(1+\frac{a_{0} \xi^{1}}{c^{2}}\right)}\right\} \cdot\left[\vec{\nabla}_{\xi}\left\{\phi_{\xi}\left(1+\frac{a_{0} \xi^{1}}{c^{2}}\right)^{2}\right\}+\frac{\partial \vec{A}_{\xi}}{c \partial \xi^{0}}\right]-\frac{1}{\left(1+\frac{a_{0} \xi^{1}}{c^{2}}\right)}\left[\nabla_{\xi}^{2}\left\{\phi_{\xi}\left(1+\frac{a_{0} \xi^{1}}{c^{2}}\right)^{2}\right\}+\frac{\partial}{c \partial \xi^{0}}\left(\vec{\nabla}_{\xi} \cdot \vec{A}_{\xi}\right)\right] \\
& =\frac{a_{0}}{c^{2}} \frac{1}{\left(1+\frac{a_{0} \xi^{1}}{c^{2}}\right)^{2}}\left[\frac{\partial}{\partial \xi^{1}}\left\{\phi_{\xi}\left(1+\frac{a_{0} \xi^{1}}{c^{2}}\right)^{2}\right\}+\frac{\partial A_{\xi^{1}}}{c \partial \xi^{0}}\right]
\end{aligned}
$$




$$
\begin{aligned}
& -\frac{1}{\left(1+\frac{a_{0} \xi^{1}}{c^{2}}\right)}\left[\nabla_{\xi}^{2}-\frac{1}{c^{2}} \frac{1}{\left(1+\frac{a_{0}}{c^{2}} \xi^{1}\right)^{2}}\left(\frac{\partial}{\partial \xi^{0}}\right)^{2}\right]\left\{\phi_{\xi}\left(1+\frac{a_{0} \xi^{1}}{c^{2}}\right)^{2}\right\} \\
& -\frac{1}{\left(1+\frac{a_{0} \xi^{1}}{c^{2}}\right)} \frac{\partial}{c \xi^{0}}\left[-\frac{1}{\left(1+\frac{a_{0} \xi^{1}}{c^{2}}\right)} \frac{A_{\xi^{1}} a_{0}}{c^{2}}\right]
\end{aligned}
$$

The Lorentz gauge in Rindler space-time is given

as

$$
\frac{1}{c} \frac{\partial \phi_{\xi}}{\partial \xi^{0}}+\vec{\nabla}_{\xi} \cdot \vec{A}_{\xi}=-\frac{1}{\left(1+\frac{a_{0} \xi^{1}}{c^{2}}\right)} \frac{a_{0}}{c^{2}} A_{\xi^{1}}
$$

Hence

$$
\begin{aligned}
& =-\frac{a_{0}}{c^{2}} \frac{1}{\left(1+\frac{a_{0} \xi^{1}}{c^{2}}\right)} E_{\xi^{1}}-\frac{1}{\left(1+\frac{a_{0} \xi^{1}}{c^{2}}\right)}\left[\nabla_{\xi^{2}}^{2}-\frac{1}{c^{2}} \frac{1}{\left(1+\frac{a_{0}}{c^{2}} \xi^{1}\right)^{2}}\left(\frac{\partial}{\partial \xi^{0}}\right)^{2}\right]\left\{\phi_{\xi}\left(1+\frac{a_{0} \xi^{1}}{c^{2}}\right)^{2}\right\}+\frac{1}{\left(1+\frac{a_{0} \xi^{1}}{c^{2}}\right)^{2}} \frac{\partial A_{\xi^{1}}}{c \partial \xi^{0}} \frac{a_{0}}{c^{2}} \\
& =4 \pi \rho_{\xi}\left(1+\frac{a_{0} \xi^{1}}{c^{2}}\right)
\end{aligned}
$$

If we apply Lorentz gauge transformation to Eqn. (46), then we have

$$
\phi_{\xi} \rightarrow \phi_{\xi}-\frac{1}{c} \frac{\partial \Lambda}{\partial \xi^{0}} \frac{1}{\left(1+\frac{a_{0} \xi^{1}}{c^{2}}\right)^{2}}, \quad \vec{A}_{\xi} \rightarrow \vec{A}_{\xi}+\vec{\nabla}_{\xi} \Lambda,
$$

Where, $\Lambda$ is a scalar function.

$$
\begin{aligned}
= & -\frac{a_{0}}{c^{2}} \frac{1}{\left(1+\frac{a_{0} \xi^{1}}{c^{2}}\right)} E_{\xi^{1}}-\frac{1}{\left(1+\frac{a_{0} \xi^{1}}{c^{2}}\right)}\left[\nabla_{\xi^{2}}^{2}-\frac{1}{c^{2}} \frac{1}{\left(1+\frac{a_{0}}{c^{2}} \xi^{1}\right)^{2}}\left(\frac{\partial}{\partial \xi^{0}}\right)^{2}\right]\left\{\phi_{\xi}\left(1+\frac{a_{0} \xi^{1}}{c^{2}}\right)^{2}\right\} \\
+ & \frac{1}{\left(1+\frac{a_{0} \xi^{1}}{c^{2}}\right)}\left[\nabla_{\xi}^{2}-\frac{1}{c^{2}} \frac{1}{\left(1+\frac{a_{0}}{c^{2}} \xi^{1}\right)^{2}}\left(\frac{\partial}{\partial \xi^{0}}\right)^{2}\right] \frac{1}{c} \frac{\partial \Lambda}{\partial \xi^{0}}+\frac{1}{\left(1+\frac{a_{0} \xi^{1}}{c^{2}}\right)^{2}} \frac{\partial A_{\xi^{1}}}{c \partial \xi^{0}} \frac{a_{0}}{c^{2}}+\frac{1}{\left(1+\frac{a_{0} \xi^{1}}{c^{2}}\right)^{2}} \frac{a_{0}}{c^{2}} \frac{\partial}{c \partial \xi^{0}} \frac{\partial \Lambda}{\partial \xi^{1}} \\
= & -\frac{a_{0}}{c^{2}} \frac{1}{\left(1+\frac{a_{0} \xi^{1}}{c^{2}}\right)} E_{\xi^{1}}-\frac{1}{\left(1+\frac{a_{0} \xi^{1}}{c^{2}}\right)}\left[\nabla_{\xi}^{2}-\frac{1}{c^{2}} \frac{1}{\left(1+\frac{a_{0}}{c^{2}} \xi^{1}\right)^{2}}\left(\frac{\partial}{\partial \xi^{0}}\right)^{2}\right]\left\{\phi_{\xi}\left(1+\frac{a_{0} \xi^{1}}{c^{2}}\right)^{2}\right\} \\
+ & \frac{1}{\left(1+\frac{a_{0} \xi^{1}}{c^{2}}\right)} \frac{\partial}{c \partial \xi^{0}}\left\{\left[\nabla_{\xi^{2}}^{2}-\frac{1}{c^{2}} \frac{1}{\left(1+\frac{a_{0}}{c^{2}} \xi^{1}\right)^{2}}\left(\frac{\partial}{\partial \xi^{0}}\right)^{2}\right] \Lambda+\frac{1}{\left(1+\frac{a_{0} \xi^{1}}{c^{2}}\right)} \frac{a_{0}}{c^{2}} \frac{\partial \Lambda}{\partial \xi^{1}}\right\}+\frac{1}{\left(1+\frac{a_{0} \xi^{1}}{c^{2}}\right)^{2}} \frac{\partial A_{\xi^{1}}}{c \partial \xi^{0}} \frac{a_{0}}{c^{2}}
\end{aligned}
$$

Now, the Lorentz gauge fixing condition, Eqn. (12) in Rindler space-time,

$$
\begin{gathered}
{\left[\frac{1}{c^{2}} \frac{1}{\left(1+\frac{a_{0} \xi^{1}}{c^{2}}\right)^{2}}\left(\frac{\partial}{\partial \xi^{0}}\right)^{2}-\nabla_{\xi}^{2}\right] \Lambda} \\
-\frac{1}{\left(1+\frac{a_{0} \xi^{1}}{c^{2}}\right)} \frac{a_{0}}{c^{2}} \frac{\partial \Lambda}{\partial \xi^{1}}=0
\end{gathered}
$$


Hence, Eqn. (44a) is

$$
\begin{aligned}
& \vec{\nabla}_{\xi} \cdot \vec{E}_{\xi}=-\frac{a_{0}}{c^{2}} \frac{1}{\left(1+\frac{a_{0} \xi^{1}}{c^{2}}\right)} E_{\xi^{1}} \\
& -\frac{1}{\left(1+\frac{a_{0} \xi^{1}}{c^{2}}\right)}\left[\nabla_{\xi}^{2}-\frac{1}{c^{2}} \frac{1}{\left(1+\frac{a_{0}}{c^{2}} \xi^{1}\right)^{2}}\left(\frac{\partial}{\partial \xi^{0}}\right)^{2}\right]\left\{\phi_{\xi}\left(1+\frac{a_{0} \xi^{1}}{c^{2}}\right)^{2}\right\} \\
& +\frac{1}{\left(1+\frac{a_{0} \xi^{1}}{c^{2}}\right)^{2}} \frac{\partial A_{\xi^{1}}}{c \partial \xi^{0}} \frac{a_{0}}{c^{2}} \\
& =4 \pi \rho_{\xi}\left(1+\frac{a_{0} \xi^{1}}{c^{2}}\right)
\end{aligned}
$$

Eqn. (44a) is invariant under Lorentz gauge transformation in Rindler space-time and Eqn. (44b) is

$$
\begin{aligned}
& \frac{1}{\left(1+\frac{a_{0} \xi^{1}}{c^{2}}\right)} \vec{\nabla}_{\xi} \times\left\{\vec{B}_{\xi}\left(1+\frac{a_{0} \xi^{1}}{c^{2}}\right)\right\} \\
& =\frac{1}{\left(1+\frac{a_{0} \xi^{1}}{c^{2}}\right)} \vec{\nabla}_{\xi} \times\left\{\vec{\nabla}_{\xi} \times \vec{A}_{\xi}\left(1+\frac{a_{0} \xi^{1}}{c^{2}}\right)\right\} \\
& =\frac{1}{\left(1+\frac{a_{0} \xi^{1}}{c^{2}}\right)} \vec{\nabla}_{\xi}\left(1+\frac{a_{0}}{c^{2}} \xi^{1}\right) \times\left\{\vec{\nabla}_{\xi} \times \vec{A}_{\xi}\right\}+\vec{\nabla}_{\xi} \times \vec{\nabla}_{\xi} \times \vec{A}_{\xi} \\
& =\frac{1}{\left(1+\frac{a_{0} \xi^{1}}{c^{2}}\right)} \frac{a_{0}}{c^{2}}(1,0,0) \times \vec{B}_{\xi}+\left\{-\nabla_{\xi}^{2} \vec{A}_{\xi}+\vec{\nabla}_{\xi}\left(\vec{\nabla}_{\xi} \cdot \vec{A}_{\xi}\right)\right\} \\
& =\frac{1}{\left(1+\frac{a_{0} \xi^{1}}{c^{2}}\right)} \frac{a_{0}}{c^{2}}\left(0,-B_{\xi^{3}}, B_{\xi^{2}}\right)+\left\{-\nabla_{\xi}^{2} \vec{A}_{\xi}+\vec{\nabla}_{\xi}\left(\vec{\nabla}_{\xi} \cdot \vec{A}_{\xi}\right)\right\} \\
& =\frac{1}{\left(1+\frac{a_{0} \xi^{1}}{c^{2}}\right)} \frac{\partial \vec{E}_{\xi}}{c \partial \xi^{0}}+\frac{4 \pi}{c} \vec{j}_{\xi} \\
& =-\frac{1}{\left(1+\frac{a_{0} \xi^{1}}{c^{2}}\right)^{2}} \frac{\partial}{c \partial \xi^{0}}\left[\vec{\nabla}_{\xi}\left\{\phi_{\xi}\left(1+\frac{a_{0} \xi^{1}}{c^{2}}\right)^{2}\right\}\right] \\
& -\frac{1}{\left(1+\frac{a_{0} \xi^{1}}{c^{2}}\right)^{2}} \frac{1}{c^{2}}\left(\frac{\partial}{\partial \xi^{0}}\right)^{2} \vec{A}_{\xi}+\frac{4 \overrightarrow{\pi j}_{\xi}}{c} \\
& =-\frac{\partial}{c \partial \xi^{0}} \vec{\nabla}_{\xi} \phi_{\xi}-\frac{1}{\left(1+\frac{a_{0} \xi^{1}}{c^{2}}\right)} \frac{2 a_{0}}{c^{2}} \frac{\partial \phi_{\xi}}{c \partial \xi^{0}}(1,0,0)
\end{aligned}
$$

$$
-\frac{1}{\left(1+\frac{a_{0} \xi^{1}}{c^{2}}\right)^{2}} \frac{1}{c^{2}}\left(\frac{\partial}{\partial \xi^{0}}\right)^{2} \vec{A}_{\xi}+\frac{4 \vec{\pi}_{\xi}}{c}
$$

Therefore,

$$
\begin{aligned}
& \frac{4 \pi}{c} \vec{j}_{\xi} \\
& =\frac{1}{\left(1+\frac{a_{0} \xi^{1}}{c^{2}}\right)} \frac{a_{0}}{c^{2}}\left(0,-B_{\xi^{3}}, B_{\xi^{2}}\right)+\left\{-\nabla_{\xi}{ }^{2} \vec{A}_{\xi}+\vec{\nabla}_{\xi}\left(\vec{\nabla}_{\xi} \cdot \vec{A}_{\xi}\right)\right\} \\
& +\frac{\partial}{c \partial \xi^{0}} \vec{\nabla}_{\xi} \phi_{\xi}+\frac{1}{\left(1+\frac{a_{0} \xi^{1}}{c^{2}}\right)} \frac{2 a_{0}}{c^{2}} \frac{\partial \phi_{\xi}}{c \partial \xi^{0}}(1,0,0) \\
& +\frac{1}{\left(1+\frac{a_{0} \xi^{1}}{c^{2}}\right)^{2}} \frac{1}{c^{2}}\left(\frac{\partial}{\partial \xi^{0}}\right)^{2} \vec{A}_{\xi} \\
& =\frac{a_{0}}{c^{2}} \frac{1}{\left(1+\frac{a_{0} \xi^{1}}{c^{2}}\right)}\left(0,-B_{\xi^{3}}, B_{\xi^{2}}\right) \\
& +\frac{1}{\left(1+\frac{a_{0}}{c^{2}} \xi^{1}\right)} \frac{2 a_{0}}{c^{2}} \frac{\partial \phi_{\xi}}{c \partial \xi^{0}}(1,0,0) \\
& +\left[-\nabla_{\xi}^{2}+\frac{1}{c^{2}} \frac{1}{\left(1+\frac{a_{0}}{c^{2}} \xi^{1}\right)^{2}}\left(\frac{\partial}{\partial \xi^{0}}\right)^{2}\right] \vec{A}_{\xi} \\
& +\vec{\nabla}_{\xi}\left[-\frac{1}{\left(1+\frac{a_{0} \xi^{1}}{c^{2}}\right)} \frac{a_{0}}{c^{2}} A_{\xi^{1}}\right] \\
& \frac{1}{c} \frac{\partial \phi_{\xi}}{\partial \xi^{0}}+\vec{\nabla}_{\xi} \cdot \vec{A}_{\xi}=-\frac{1}{\left(1+\frac{a_{0} \xi^{1}}{c^{2}}\right)} \frac{a_{0}}{c^{2}} A_{\xi^{1}} \\
& \frac{4 \pi}{c} \vec{j}_{\xi} \\
& =\frac{a_{0}}{c^{2}} \frac{1}{\left(1+\frac{a_{0} \xi^{1}}{c^{2}}\right)}\left(0,-B_{\xi^{3}}, B_{\xi^{2}}\right) \\
& +\frac{1}{\left(1+\frac{a_{0}}{c^{2}} \xi^{1}\right)} \frac{2 a_{0}}{c^{2}} \frac{\partial \phi_{\xi}}{c \partial \xi^{0}}(1,0,0) \\
& +\left[-\nabla_{\xi}^{2}+\frac{1}{c^{2}} \frac{1}{\left(1+\frac{a_{0}}{c^{2}} \xi^{1}\right)^{2}}\left(\frac{\partial}{\partial \xi^{0}}\right)^{2}\right] \vec{A}_{\xi} \\
& +\vec{\nabla}_{\xi}\left[-\frac{1}{\left(1+\frac{a_{0} \xi^{1}}{c^{2}}\right)} \frac{a_{0}}{c^{2}} A_{\xi^{1}}\right]
\end{aligned}
$$


If we apply the Lorentz gauge transformation to Eqn. (52), we get

$$
\phi_{\xi} \rightarrow \phi_{\xi}-\frac{1}{c} \frac{\partial \Lambda}{\partial \xi^{0}} \frac{1}{\left(1+\frac{a_{0} \xi^{1}}{c^{2}}\right)^{2}} \quad, \quad \vec{A}_{\xi} \rightarrow \vec{A}_{\xi}+\vec{\nabla}_{\xi} \Lambda
$$

Where, $\Lambda$ is a scalar function.

$$
\begin{aligned}
& \frac{4 \pi}{c} \vec{j}_{\xi}=\frac{a_{0}}{c^{2}} \frac{1}{\left(1+\frac{a_{0} \xi^{1}}{c^{2}}\right)}\left(0,-B_{\xi^{3}}, B_{\xi^{2}}\right)+\frac{1}{\left(1+\frac{a_{0}}{c^{2}} \xi^{1}\right)} \frac{2 a_{0}}{c^{2}} \frac{\partial \phi_{\xi}}{c \partial \xi^{0}}(1,0,0)-\frac{1}{\left(1+\frac{a_{0}}{c^{2}} \xi^{1}\right)^{3}} \frac{2 a_{0}}{c^{2}} \frac{1}{c^{2}}\left(\frac{\partial}{\partial \xi^{0}}\right)^{2} \Lambda(1,0,0) \\
& +\left[-\nabla_{\xi}^{2}+\frac{1}{c^{2}} \frac{1}{\left(1+\frac{a_{0}}{c^{2}} \xi^{1}\right)^{2}}\left(\frac{\partial}{\partial \xi^{0}}\right)^{2}\right] \vec{A}_{\xi}+\left[-\nabla_{\xi}^{2}+\frac{1}{c^{2}} \frac{1}{\left(1+\frac{a_{0}}{c^{2}} \xi^{1}\right)^{2}}\left(\frac{\partial}{\partial \xi^{0}}\right)^{2}\right] \vec{\nabla}_{\xi} \Lambda \\
& +\vec{\nabla}_{\xi}\left[-\frac{1}{\left(1+\frac{a_{0} \xi^{1}}{c^{2}}\right)} \frac{a_{0}}{c^{2}} A_{\xi^{1}}\right]+\vec{\nabla}_{\xi}\left[-\frac{1}{\left(1+\frac{a_{0} \xi^{1}}{c^{2}}\right)} \frac{a_{0}}{c^{2}} \frac{\partial \Lambda}{\partial \xi^{1}}\right] \\
& =\frac{a_{0}}{c^{2}} \frac{1}{\left(1+\frac{a_{0} \xi^{1}}{c^{2}}\right)}\left(0,-B_{\xi^{3}}, B_{\xi^{2}}\right)+\frac{1}{\left(1+\frac{a_{0}}{c^{2}} \xi^{1}\right)} \frac{2 a_{0}}{c^{2}} \frac{\partial \phi_{\xi}}{c \partial \xi^{0}}(1,0,0)-\frac{1}{\left(1+\frac{a_{0}}{c^{2}} \xi^{1}\right)^{3}} \frac{2 a_{0}}{c^{2}} \frac{1}{c^{2}}\left(\frac{\partial}{\partial \xi^{0}}\right)^{2} \Lambda(1,0,0) \\
& +\left[-\nabla_{\xi}^{2}+\frac{1}{c^{2}} \frac{1}{\left(1+\frac{a_{0}}{c^{2}} \xi^{1}\right)^{2}}\left(\frac{\partial}{\partial \xi^{0}}\right)^{2}\right] \vec{A}_{\xi}+\left[-\nabla_{\xi}^{2}+\frac{1}{c^{2}} \frac{1}{\left(1+\frac{a_{0}}{c^{2}} \xi^{1}\right)^{2}}\left(\frac{\partial}{\partial \xi^{0}}\right)^{2}\right] \vec{\nabla}_{\xi} \Lambda \\
& +\frac{1}{\left(1+\frac{a_{0} \xi^{1}}{c^{2}}\right)^{2}} \frac{a_{0}^{2}}{c^{4}} A_{\xi^{1}}(1,0,0)-\frac{1}{\left(1+\frac{a_{0} \xi^{1}}{c^{2}}\right)} \frac{a_{0}}{c^{2}} \vec{\nabla}_{\xi} A_{\xi^{1}} \\
& +\frac{1}{\left(1+\frac{a_{0} \xi^{1}}{c^{2}}\right)^{2}} \frac{a_{0}^{2}}{c^{4}} \frac{\partial \Lambda}{\partial \xi^{1}}(1,0,0)-\frac{1}{\left(1+\frac{a_{0} \xi^{1}}{c^{2}}\right)} \frac{a_{0}}{c^{2}} \frac{\partial}{\partial \xi^{1}} \vec{\nabla}_{\xi} \Lambda
\end{aligned}
$$

Now, the Lorentz gauge fixing condition, Eqn. (12) in Rindler space-time, is

$$
\begin{aligned}
& {\left[\frac{1}{c^{2}} \frac{1}{\left(1+\frac{a_{0} \xi^{1}}{c^{2}}\right)^{2}}\left(\frac{\partial}{\partial \xi^{0}}\right)^{2}-\nabla_{\xi}^{2}\right] \Lambda-\frac{1}{\left(1+\frac{a_{0} \xi^{1}}{c^{2}}\right)} \frac{a_{0}}{c^{2}} \frac{\partial \Lambda}{\partial \xi^{1}}=0 } \\
& 0=\vec{\nabla}_{\xi}\left[\left\{-\nabla_{\xi}^{2}+\frac{1}{c^{2}} \frac{1}{\left(1+\frac{a_{0}}{c^{2}} \xi^{1}\right)^{2}}\left(\frac{\partial}{\partial \xi^{0}}\right)^{2}-\frac{1}{\left(1+\frac{a_{0} \xi^{1}}{c^{2}}\right)} \frac{a_{0}}{c^{2}} \frac{\partial}{\partial \xi^{1}}\right\} \Lambda\right] \\
&=\vec{\nabla}_{\xi}\left\{\frac{1}{c^{2}} \frac{1}{\left(1+\frac{a_{0}}{c^{2}} \xi^{1}\right)^{2}}\right\}\left(\frac{\partial}{\partial \xi^{0}}\right)^{2} \Lambda+\left[-\nabla_{\xi}^{2}+\frac{1}{c^{2}} \frac{1}{\left(1+\frac{a_{0}}{c^{2}} \xi^{1}\right)^{2}}\left(\frac{\partial}{\partial \xi^{0}}\right)^{2}\right] \vec{\nabla}_{\xi} \Lambda \\
& \quad-\vec{\nabla}_{\xi}\left\{\frac{a_{0}}{c^{2}} \frac{1}{\left(1+\frac{a_{0}}{c^{2}} \xi^{1}\right)}\right\} \frac{\partial \Lambda}{\partial \xi^{1}}-\frac{1}{\left(1+\frac{a_{0}}{c^{2}} \xi^{1}\right)} \frac{a_{0}}{c^{2}} \frac{\partial}{\partial \xi^{1}} \vec{\nabla}_{\xi} \Lambda \\
&=-\frac{2}{c^{2}} \frac{1}{\left(1+\frac{a_{0}}{c^{2}} \xi^{1}\right)^{3}} \frac{a_{0}}{c^{2}}\left(\frac{\partial}{\partial \xi^{0}}\right)^{2} \Lambda(1,0,0)+\left[-\nabla_{\xi}^{2}+\frac{1}{c^{2}} \frac{1}{\left(1+\frac{a_{0}}{c^{2}} \xi^{1}\right)^{2}}\left(\frac{\partial}{\partial \xi^{0}}\right)^{2}\right] \vec{\nabla}_{\xi} \Lambda
\end{aligned}
$$




$$
+\frac{a_{0}{ }^{2}}{c^{4}} \frac{1}{\left(1+\frac{a_{0}}{c^{2}} \xi^{1}\right)^{2}} \frac{\partial \Lambda}{\partial \xi^{1}}(1,0,0)-\frac{1}{\left(1+\frac{a_{0}}{c^{2}} \xi^{1}\right)} \frac{a_{0}}{c^{2}} \frac{\partial}{\partial \xi^{1}} \vec{\nabla}_{\xi} \Lambda
$$

Therefore, Eqn. (53) is

$$
\begin{aligned}
& \frac{4 \pi}{c} \vec{j}_{\xi}=\frac{a_{0}}{c^{2}} \frac{1}{\left(1+\frac{a_{0} \xi^{1}}{c^{2}}\right)}\left(0,-B_{\xi^{3}}, B_{\xi^{2}}\right)+\frac{1}{\left(1+\frac{a_{0}}{c^{2}} \xi^{1}\right)} \frac{2 a_{0}}{c^{2}} \frac{\partial \phi_{\xi}}{c \partial \xi^{0}}(1,0,0) \\
& -\frac{1}{\left(1+\frac{a_{0}}{c^{2}} \xi^{1}\right)^{3}} \frac{2 a_{0}}{c^{2}} \frac{1}{c^{2}}\left(\frac{\partial}{\partial \xi^{0}}\right)^{2} \Lambda(1,0,0)+\left[-\nabla_{\xi^{2}}{ }^{2}+\frac{1}{c^{2}} \frac{1}{\left(1+\frac{a_{0}}{c^{2}} \xi^{1}\right)^{2}}\left(\frac{\partial}{\partial \xi^{0}}\right)^{2}\right] \vec{A}_{\xi}+\frac{1}{\left(1+\frac{a_{0} \xi^{1}}{c^{2}}\right)^{2}} \frac{a_{0}{ }^{2}}{c^{4}} A_{\xi^{1}}(1,0,0) \\
& -\frac{1}{\left(1+\frac{a_{0} \xi^{1}}{c^{2}}\right)} \frac{a_{0}}{c^{2}} \vec{\nabla}_{\xi} A_{\xi^{1}}+\frac{1}{\left(1+\frac{a_{0} \xi^{1}}{c^{2}}\right)^{2}} \frac{a_{0}^{2}}{c^{4}} \frac{\partial \Lambda}{\partial \xi^{1}}(1,0,0)-\frac{1}{\left(1+\frac{a_{0} \xi^{1}}{c^{2}}\right)} \frac{a_{0}}{c^{2}} \frac{\partial}{\partial \xi^{1}} \vec{\nabla}_{\xi} \Lambda \\
& +\vec{\nabla}_{\xi}\left[\left\{-\nabla_{\xi}^{2}+\frac{1}{c^{2}} \frac{1}{\left(1+\frac{a_{0}}{c^{2}} \xi^{1}\right)^{2}}\left(\frac{\partial}{\partial \xi^{0}}\right)^{2}-\frac{1}{\left(1+\frac{a_{0} \xi^{1}}{c^{2}}\right)} \frac{a_{0}}{c^{2}} \frac{\partial}{\partial \xi^{1}}\right\} \Lambda\right] \\
& +\frac{2}{c^{2}} \frac{1}{\left(1+\frac{a_{0}}{c^{2}} \xi^{1}\right)^{3}} \frac{a_{0}}{c^{2}}\left(\frac{\partial}{\partial \xi^{0}}\right)^{2} \Lambda(1,0,0)-\frac{a_{0}^{2}}{c^{4}} \frac{1}{\left(1+\frac{a_{0}}{c^{2}} \xi^{1}\right)^{2}} \frac{\partial \Lambda}{\partial \xi^{1}}(1,0,0)+\frac{1}{\left(1+\frac{a_{0}}{c^{2}} \xi^{1}\right)} \frac{a_{0}}{c^{2}} \frac{\partial}{\partial \xi^{1}} \vec{\nabla}_{\xi} \Lambda \\
& =\frac{a_{0}}{c^{2}} \frac{1}{\left(1+\frac{a_{0} \xi^{1}}{c^{2}}\right)}\left(0,-B_{\xi^{3}}, B_{\xi^{2}}\right)+\frac{1}{\left(1+\frac{a_{0}}{c^{2}} \xi^{1}\right)} \frac{2 a_{0}}{c^{2}} \frac{\partial \phi_{\xi}}{c \partial \xi^{0}}(1,0,0) \quad+\left[-\nabla_{\xi}^{2}+\frac{1}{c^{2}} \frac{1}{\left(1+\frac{a_{0}}{c^{2}} \xi^{1}\right)^{2}}\left(\frac{\partial}{\partial \xi^{0}}\right)^{2}\right] \vec{A}_{\xi} \\
& +\frac{1}{\left(1+\frac{a_{0} \xi^{1}}{c^{2}}\right)^{2}} \frac{a_{0}^{2}}{c^{4}} A_{\xi^{1}}(1,0,0)-\frac{1}{\left(1+\frac{a_{0} \xi^{1}}{c^{2}}\right)} \frac{a_{0}}{c^{2}} \vec{\nabla}_{\xi^{\prime}} A_{\xi^{1}}
\end{aligned}
$$

Hence, Eqn. (44b) is invariant under Lorentz gauge transformation in Rindler space-time.

Now, Eqn. (44c) is

$$
\vec{\nabla}_{\xi} \cdot \vec{B}_{\xi}=\vec{\nabla}_{\xi} \cdot\left(\vec{\nabla}_{\xi} \times \vec{A}_{\xi}+\vec{\nabla}_{\xi} \times \vec{\nabla}_{\xi} \Lambda\right)=\vec{\nabla}_{\xi} \times \vec{\nabla}_{\xi} \cdot \vec{A}_{\xi}=0
$$

And, Eqn. (44d) is

$$
\begin{aligned}
& \frac{1}{\left(1+\frac{a_{0} \xi^{1}}{c^{2}}\right)} \vec{\nabla}_{\xi} \times\left\{\vec{E}_{\xi}\left(1+\frac{a_{0} \xi^{1}}{c^{2}}\right)\right\} \\
& =-\frac{1}{\left(1+\frac{a_{0} \xi^{1}}{c^{2}}\right)} \vec{\nabla}_{\xi} \times\left[\vec{\nabla}_{\xi}\left\{\phi_{\xi}\left(1+\frac{a_{0} \xi^{1}}{c^{2}}\right)^{2}\right\}-\vec{\nabla}_{\xi}\left(\frac{\partial \Lambda}{c \partial \xi^{0}}\right)+\frac{\partial \vec{A}_{\xi}}{c \partial \xi^{0}}+\frac{\partial}{c \partial \xi^{0}}\left(\vec{\nabla}_{\xi} \Lambda\right)\right] \\
& =-\frac{1}{\left(1+\frac{a_{0} \xi^{1}}{c^{2}}\right)} \vec{\nabla}_{\xi} \times \frac{\partial \vec{A}_{\xi}}{c \partial \xi^{0}}=-\frac{1}{\left(1+\frac{a_{0} \xi^{1}}{c^{2}}\right)} \frac{\partial\left(\vec{\nabla}_{\xi} \times \vec{A}_{\xi}\right)}{c \partial \xi^{0}}=-\frac{1}{\left(1+\frac{a_{0} \xi^{1}}{c^{2}}\right)} \frac{\partial \vec{B}_{\xi}}{c \partial \xi^{0}}
\end{aligned}
$$

Hence, Eqn. (44c) and Eqn. (44d) are invariant under Lorentz gauge transformation in Rindler space-time. Hence, the electromagnetic field equations (Maxwell Equations) in Rindler space- time, Eqns. (44a)-(44d) are also invariant under Lorentz gauge transformation. 


\section{Conclusion}

Since del Castillio and Sanchez already calculated Maxwell equations in uniformly accelerated frame in vacuum, and Maluf and Faria obtained electromagnetic field transformation in Rindler space-time [5] (see ArXiv preprint), we computed the electromagnetic field transformation and the electro-magnetic equation in a uniformly accelerated frame in a single theory.

Generally, the coordinate transformation of accelerated frame (see Ref. [6]) is

(I) $c t=\left(\frac{c^{2}}{a_{0}}+\xi^{1}\right) \sinh \left(\frac{a_{0} \xi^{0}}{c}\right)$

$$
x=\left(\frac{c^{2}}{a_{0}}+\xi^{1}\right) \cosh \left(\frac{a_{0} \xi^{0}}{c}\right)-\frac{c^{2}}{a_{0}}, y=\xi^{2}, z=\xi^{3}
$$

(II) $c t=\frac{c^{2}}{a_{0}} \exp \left(\frac{a_{0}}{c^{2}} \xi^{1}\right) \sinh \left(\frac{a_{0} \xi^{0}}{c}\right)$

$$
\begin{aligned}
E_{\xi^{1}} & =E_{x}=E_{X 0} \sin \omega\left(t-\frac{x}{c}\right) \\
& =E_{x 0} \sin \omega\left[-\left(\left\{\frac{c}{a_{0}}+\frac{\xi^{1}}{c}\right\} \exp \left(-\frac{a_{0}}{c} \xi^{0}\right)+\frac{c}{a_{0}}\right)\right]=E_{x 0} \sin \Phi \\
c t & =\left(\frac{c^{2}}{a_{0}}+\xi^{1}\right) \sinh \left(\frac{a_{0} \xi^{0}}{c}\right), \quad x=\left(\frac{c^{2}}{a_{0}}+\xi^{1}\right) \cosh \left(\frac{a_{0} \xi^{0}}{c}\right)-\frac{c^{2}}{a_{0}}
\end{aligned}
$$

Now,

$$
-\exp \left(-\frac{a_{0}}{c} \xi^{0}\right)=\sinh \left(\frac{a_{0} \xi^{0}}{c}\right)-\cosh \left(\frac{a_{0} \xi^{0}}{c}\right)
$$

(A1) must satisfy the following equation.

$$
\begin{aligned}
\frac{1}{\left(1+\frac{a_{0} \xi^{1}}{c}\right)^{2}} \frac{1}{c^{2}}\left(\frac{\partial}{\partial \xi^{0}}\right)^{2} \sin \Phi=\left(\frac{\partial}{\partial \xi^{1}}\right)^{2} \sin \Phi \\
\left(\frac{\partial}{\partial \xi^{1}}\right)^{2} \sin \Phi=\frac{\partial}{\partial \xi^{1}}\left\{\frac{\partial}{\partial \xi^{1}} \sin \omega\left[-\left\{\frac{c}{a_{0}}+\frac{\xi^{1}}{c}\right\} \exp \left(-\frac{a_{0}}{c} \xi^{0}\right)+\frac{c}{a_{0}}\right]\right\} \\
=\frac{\partial}{\partial \xi^{1}}\left\{(\cos \Phi) \cdot-\omega \frac{1}{c} \exp \left(-\frac{a_{0}}{c} \xi^{0}\right)\right\}=(-\sin \Phi) \cdot \frac{\omega^{2}}{c^{2}} \exp \left(-2 \frac{a_{0}}{c} \xi^{0}\right)
\end{aligned}
$$

But this computational situation is different.

$$
\frac{1}{\left(1+\frac{a_{0} \xi^{1}}{c}\right)^{2}} \frac{1}{c^{2}}\left(\frac{\partial}{\partial \xi^{0}}\right)^{2} \sin \Phi=\frac{1}{\left(1+\frac{a_{0} \xi^{1}}{c^{2}}\right)^{2}} \frac{1}{c^{2}} \frac{\partial}{\partial \xi^{0}}\left\{\frac{\partial}{\partial \xi^{0}} \sin \omega\left[-\left\{\frac{c}{a_{0}}+\frac{\xi^{1}}{c}\right\} \exp \left(-\frac{a_{0}}{c} \xi^{0}\right)+\frac{c}{a_{0}}\right]\right\}
$$




$$
=\frac{1}{\left(1+\frac{a_{0} \xi^{1}}{c^{2}}\right)^{2}} \frac{1}{c^{2}} \frac{\partial}{\partial \xi^{0}}\left\{(\cos \Phi) \cdot \omega\left\{\frac{c}{a_{0}}+\frac{\xi^{1}}{c}\right\} \frac{a_{0}}{c} \exp \left(-\frac{a_{0}}{c} \xi^{0}\right)\right\}
$$

Now, $D(\alpha \beta)=\beta D \alpha+\alpha D \beta$

$$
\begin{aligned}
= & \frac{1}{\left(1+\frac{a_{0} \xi^{1}}{c^{2}}\right)^{2}} \frac{1}{c^{2}}\left\{\frac{\partial}{\partial \xi^{0}}(\cos \Phi) \cdot \omega\left\{\frac{c}{a_{0}}+\frac{\xi^{1}}{c}\right\} \frac{a_{0}}{c} \exp \left(-\frac{a_{0}}{c} \xi^{0}\right) \quad+(\cos \Phi) \cdot \omega\left\{\frac{c}{a_{0}}+\frac{\xi^{1}}{c}\right\} \frac{a_{0}}{c} \frac{\partial}{\partial \xi^{0}}\left(\exp \left(-\frac{a_{0}}{c} \xi^{0}\right)\right)\right\} \\
= & \frac{1}{\left(1+\frac{a_{0} \xi^{1}}{c^{2}}\right)^{2}} \frac{1}{c^{2}}\left\{-\sin \Phi \cdot \omega^{2}\left\{\frac{c}{a_{0}}+\frac{\xi^{1}}{c}\right\}^{2} \frac{a_{0}^{2}}{c^{2}} \exp \left(-2 \frac{a_{0}}{c} \xi^{0}\right)-(\cos \Phi) \cdot \omega\left\{\frac{c}{a_{0}}+\frac{\xi^{1}}{c}\right\} \frac{a_{0}{ }^{2}}{c^{2}} \exp \left(-\frac{a_{0}}{c} \xi^{0}\right)\right\}
\end{aligned}
$$

Hence, if we compare Eqn. (A4) and Eqn. (A5),

$$
\begin{aligned}
\left(\frac{\partial}{\partial \xi^{1}}\right)^{2} \sin \Phi= & (-\sin \Phi) \cdot \frac{\omega^{2}}{c^{2}} \exp \left(-2 \frac{a_{0}}{c} \xi^{0}\right) \neq-\sin \Phi \cdot \omega^{2} \frac{1}{c^{2}} \exp \left(-2 \frac{a_{0}}{c} \xi^{0}\right) \\
& -\frac{1}{\left(1+\frac{a_{0} \xi^{1}}{c^{2}}\right)}(\cos \Phi) \cdot \omega \frac{a_{0}}{c} \frac{1}{c^{2}} \exp \left(-\frac{a_{0}}{c} \xi^{0}\right)=\frac{1}{\left(1+\frac{a_{0} \xi^{1}}{c}\right)^{2}} \frac{1}{c^{2}}\left(\frac{\partial}{\partial \xi^{0}}\right)^{2} \sin \Phi
\end{aligned}
$$

We conclude that it cannot exist as the electromagnetic wave function in Rindler spacetime.

\section{References}

[1] G. F. Torres del Castillio and C. I. Perez Sanchez, Revista Mexicana De Fisica 53, 1,4 (2007).

[2] J. W. Maluf and F. F. Faria, "The electromagnetic field in accelerated frames", Arxiv:gr-qc/1110.5367v1 (2011).

[3] W. Rindler, Am. J. Phys. 34, 1174 (1966).

[4] S. Yi, The African Review of Physics 8, 37 (2013).

[5] F. Shojai and A. Shojai, "The equivalence principle and the relativity velocity of local inertial frame", Arxiv:gr-qc/1505.06691v1 (2015).

[6] Massimo Pauri and Michele Vallisner, "Marzke-Wheeler coordinates for accelerated observers in special relativity", Arxiv:gr-qc/0006095 (2000).

[7] A. Einstein, "Zur Elektrodynamik bewegter Körper”, Annalen der Physik 17, 891 (1905).

Received: 6 October, 2016 Accepted: 25 November, 2016 